\title{
Role of Hydrogen Peroxide in Improving Potato Tuber Quality
}

\author{
Sameh A.M. Moussa ${ }^{1}$, Nashwa I. Abo El-Fadl ${ }^{1}$ and Neveen F. Agamy ${ }^{2}$
}

\begin{abstract}
Four potato cultivars were planted during the summer seasons of 2009 and 2010 at Sabaheya Horticultural Research Station, Alexandria governorate, Egypt. The experiments were conducted to study the effect of hydrogen peroxide in increasing the proportion of dry matter in potato tubers which is very important in the production of chips and French fries. The growing plants were sprayed twice a week with hydrogen peroxide in four concentrations (zero, 20, 40 and $60 \mathrm{mM}$ ). Tubers' starch percentage was significantly increased with increasing the hydrogen peroxide up to $60 \mathrm{mM}$. The highest percentage of tubers' dry matter was possessed when plants was sprayed with $40 \mathrm{mM}$ of hydrogen peroxide. Parenchyma cell diameter was affected with hydrogen peroxide concentrations, where, the data detected that the ratio of increasing in tuber parenchyma cell diameter reached $9.34 \%$ as compared to with non-treated plants. It could be said that spraying hydrogen peroxide on potato plants at the rate of $40 \mathrm{mM}$ had positive effect on potato yield and tubers' dry matter percentage. Sensory evaluation for potato French fries and chips showed that zero and $20 \mathrm{mM}$ of hydrogen peroxide treatments possessed the highest acceptability of the produced French fries and chips. Anushka and Universal potato cultivars were the best for producing French fries at the levels of zero, 20 and $40 \mathrm{mM}$ hydrogen peroxide, while Dora cultivar was elected for producing potato chips at all hydrogen peroxide concentrations.
\end{abstract}

Key words: potato, Solanum tuberosum, L., hydrogen peroxide, parenchyma cells, starch accumulation, potato processing, French frying and potato chips.

\section{INTRODUCTION}

Potatoes (Solanum tuberosum, L.) are considered one of the most important vegetable crops which are grown in large areas in Egypt, where the total area planted with potatoes, according to estimates of the Egyptian Ministry of Agriculture for the year 2012 about 200 thousands feddan spread over three lugs (summer, Nili and winter) with a total production reached more than 2 million tons, with an average productivity about 10 tons per feddan. In the past ten years, processing potatoes was increased in Egypt, both for the production of potato chips or for French fries. These processes require a number of specifications to keep the products in high quality. Among the most important factors is the high net product ratio which is highly associated with the percentage of dry matter and starch, in addition to the lack of reducing sugars._Many researches are directed to increase the quality processing. It has been suggested that foliar application of salicylate could enhance tuber industrial quality by increasing dry matter and starch content (Nickell 1991). Tuber starch is an important quality character for potato crops. Dry matter is generally used as an index of starch content by growers and breeders and it is an important factor to decide the destination of the produce (Gould and Plimpton 1985; Estrada 2000). Tubers with high dry matter content, for example, require less energy and absorb less oil during frying, and have a drier texture after cooking (Storey and Davies 1992). For industrial purposes, millions of tones of starch and dextrins are produced and modified annually from potato tubers, with yields proportional to the starch content of the raw material (Burton 1989). Delgado et al. (2005) demonstrated in their research that field plants treated with 5 or $50 \mathrm{mM}$ hydrogen peroxide significantly enhanced tuber starch accumulation by between $6.7 \%$ and $30 \%$, respectively, and they found that hydrogen peroxide treated stems were up to $27 \%$ thicker than controls, and explained that this result mainly due to enlarged medullar parenchyma cells. Moreover, quantification using image analysis confirmed that stems of hydrogen peroxide treated plants contained up to 3.4-fold more starch and $62 \%$ more lignin. This new chemical treatment to promote starch accumulation has potential utility in potato crop production and research (Delgado et al., 2005).

This research aimed to improve the quality of processing potatoes and to increase the net products by increasing dry matter and starch percentages of potato tubers, and this will encourage further extension in processes.

\section{MATERIALS AND METHODS}

The present investigation was carried out during the two summer seasons of 2009 and 2010 at Sabaheya Horticultural Research Station, Alexandria governorate, Egypt. Planting tuber seeds toke place on the middle of January in both growing seasons; using four potato cultivars; Anushka, Universal, Vivaldi and Dora. Tuber seeds were planted in rows, $60 \mathrm{~cm}$ in wide, 4.0 long and at spacing of $25 \mathrm{~cm}$ within rows.

\section{Treatments}

Each experiment consisted of four cultivars representing the main plots. Each cultivar was grown in sixteen rows. Four concentrations of hydrogen peroxide $\left(\mathrm{H}_{2} \mathrm{O}_{2}\right)(60 \mathrm{mM}, 40 \mathrm{mM}, 20 \mathrm{mM}$ and zero $\mathrm{mM})$ were randomly distributed in the sub-plots. Each sub-plot consisted of four rows having an area of $9.60 \mathrm{~m}^{2}$. Hydrogen peroxide was sprayed to the plants twice

\footnotetext{
${ }^{1}$ Sabaheya Horticultural Research station, Horticultural Research Institute, Agricultural Research Center, Egypt.

${ }^{2}$ Nutrition Department, Food Analysis Division,

High Institute of Public Health, Alexandria University.

E-mail address for correspondence: samehmoussa@yahoo.com

Received May20, 2012, Accepted June 28,, 2012
} 
Table 1. Physical properties and chemical analyses of the experimental soil

\begin{tabular}{|c|c|c|c|c|c|c|c|c|c|}
\hline \multicolumn{10}{|c|}{ Soil analyses of Sabaheya Farm } \\
\hline \multicolumn{3}{|c|}{ Mechanical analysis } & \multirow{2}{*}{\multicolumn{3}{|c|}{ Texture }} & \multirow{2}{*}{ pH } & \multirow{2}{*}{ EC. dS/m } & \multirow{2}{*}{$\begin{array}{c}\mathrm{CaCo}_{3} \\
\%\end{array}$} & \multirow{2}{*}{$\begin{array}{c}\text { O.M. } \\
\%\end{array}$} \\
\hline Sand $\%$ & Silt \% & Clay \% & & & & & & & \\
\hline 23.90 & 42.50 & 33.60 & & clay sl & & 8.33 & 1.8 & 22.00 & 0.57 \\
\hline \multicolumn{10}{|c|}{ Chemical analysis } \\
\hline \multicolumn{6}{|c|}{ Cations (meq/L) } & \multicolumn{4}{|c|}{ Anions (meq/L) } \\
\hline $\mathbf{N}^{+}$ & $\mathbf{P}^{+}$ & $\mathrm{Ca}^{++}$ & $\mathrm{Mg}^{++}$ & $\mathbf{N a}^{+}$ & $\mathbf{K}^{+}$ & $\mathrm{CO}_{3}^{-}$ & $\mathrm{HCO}_{3}^{-}$ & $\mathbf{C L}^{-}$ & $\mathrm{SO}_{4}^{--}$ \\
\hline 27.5 & 19.7 & 0.94 & 0.39 & 081 & 895.0 & zero & 1.83 & 0.45 & 2.00 \\
\hline
\end{tabular}

a week at 40 days from planting up to 90 days. The spraying was carried out early in the morning until plants were dipping wet with a hand pressure sprayer. The two central rows of each sup-plot were used for analytical samples. The physical and chemical analyses of the experimental soil are presented in table, 1. All the agricultural practices used for commercial potato production, as common in this area, were carried out in both years

\section{Measurements}

Vegetative growth and yield parameters: Ten whole plant samples per sub-plot were randomly used, 90 days after planting, for the determination of the vegetative growth (plant height $(\mathrm{cm})$ and number of branches). Stem diameter was measured using a caliper on the fourth internodes from the base. A certain weight of fresh foliage of five plants, fro each sub-plot, was dried and the percentage of dry weight was recorded. Another Ten random plants were used at harvest (110 days) to determine plant tuber yield $(\mathrm{kg})$. Tuber yield was determined in weight and number of all tubers per plant.

Physical characteristics: Random samples of 20 tubers per treatment were randomly used to measure the physical characteristics of the tubers; tuber length and diameter were measured to calculate the tuber shape index by dividing the former by the latter. Tuber specific gravity was determined by weighting a certain weight of tubers for each treatment, then the specific gravity was computed according to the following equation:-

Tuber' weight in air

Tuber specific gravity $=$

Tuber' weight in air - Tuber' weight in water

Tuber quality: Random samples of 10 tubers per treatment for each replicate were randomly used to determine the tuber quality characters.

1- Tuber dry matter (\%): Was carried out by weighing a certain weight of fresh tubers and then dried.

Dry weight

Dry matter \% = ----------- X 100

Fresh weight
2- Determination of reducing and non-reducing sugars percentages $(\%)$ : A known mass $(5 \mathrm{~g})$ of fresh tuber was taken to determine reducing and nonreducing sugars, using sulphuric acid and phenol (5\%); then they were colourimetrically determined, according to the method of Dubios et. al. (1956).

3- Determination of starch: Tuber starch percentage (\%) was determined using a sample of $1 \mathrm{~g}$ of fresh tuber, according to the method described in A.O.A.C. (1970).

Histology examination (in the second season only): Five random tubers were taken from each treatment in each replicate and then peeled and cut with a sharp cork on sliding microtome into slices $1 \mathrm{~mm}$ thickness and $10 \mathrm{~mm}$ in diameter. The potato slices were mixed and washed with distilled water. Ten randomly slices were taken for examination under a compound microscope using a high power (400 X) objective to determine the parenchyma cells diameter; in micron $(\mu)$, of potato tubers.

\section{Evaluation of potato chips and strips processed}

Potatoes (four different cultivars of potatoes, each cultivar which was treated with one of four concentrations of $\mathrm{H}_{2} \mathrm{O}_{2}$ in two cultivations) and vegetable oil (blend of cottonseed and sun flower with 1:1 ratio) were the raw materials. Potato tubers were stored at $8{ }^{\circ} \mathrm{C}$ and $95 \%$ relative humidity. Chips (Slices) (thickness of $3 \mathrm{~mm}$ ) were cut from the pith of the parenchymatous region of potato tubers using an electric slicing machine (Berkel, model EAS65, UK). Strips were prepared by cutting tubers into strips with base dimension $10 \times 10 \mathrm{~mm}^{2}$ and different lengths using an electric machine (Berkel, model EAS65, UK)

\section{1-Blanching}

Chips and strips were rinsed for $1 \mathrm{~min}$ in distilled water, immediately after cutting, to eliminate some starch adhering to the surface prior to frying. All samples were kept between humidified paper towels to avoid excessive dehydration before frying. 


\section{2-Frying experiments}

The frying time was constant ( $3 \mathrm{~min})$. Twenty slices or strips per sampling time were deep-fried in hot oil contained in an 3 Liter capacity electrical fryer (Rival, Model CZF575, China) at a temperature $\left(180 \pm 1^{\circ} \mathrm{C}\right)$.

\section{3-Determination of the dry matter}

The raw material was determined for dry matter by drying method at a temperature of $105{ }^{\circ} \mathrm{C}$ (A.O.A.C., 1995). The dry matter was also found to be the ratio of the weight of samples after frying and its weight before frying multiplied by 100 .

\section{4-Sensory evaluation}

The sensory evaluation was carried out on the processed chips and strips using trained panelists (Staff members of the Department of Nutrition, High Institute of Public Health, Alexandria University) which are frequently used to do such test. Each panelist recorded the results in special sheet using 7-point descriptive category scale for each estimated parameter, in which one indicating very poor and 7 indicates excellent.

\section{Experimental design and statistical analysis}

The used experimental layout was arranged as a split-plot in a randomized complete blocks design (R.C.B.D), with three replicates. Four potato cultivars were considered as main plots, and four concentrations of hydrogen peroxide were randomly sprayed in the supplots. Collected data of the experiments were statistically analyzed, using the analysis of variance method. Comparisons among the means of different treatments were done, using Duncan's multiple range test procedure at $\mathrm{p}=0.05$ level of significance, as illustrated by Snedecor and Cochran (1980). Computation was done using SAS (2001).

\section{RESULTS AND DISCUSSION}

\section{Results and Discussion}

\section{A. Potato morphological characters, tuber yield and quality attributes}

\section{A.1. Morphological characters}

Data presented in table, 2, clearly, showed the existence of significant differences among the examined cultivars for most of studied vegetative traits over the two years with the exception of foliage dry matter during first year of the study. Data of table, 2 Impact the concentrations of hydrogen peroxide on the studied vegetative traits. It became clear from these data that there was no significant effect of the concentrations of hydrogen peroxide on plant height during the two years of study, as well as there were no effects of these concentrations of hydrogen on the number of branches per plant in first year of experiment. The results also showed that the concentrations 40 and $60 \mathrm{mM}$ of hydrogen peroxide gave the best results compared to other concentrations for percentage of foliage dry matter and stem diameter during the two seasons. Mean diameter of sixth internodes of $\mathrm{H}_{2} \mathrm{O}_{2}$ treated plants was $27 \%(5 \mathrm{mM})$ and $21 \%(50 \mathrm{mM})$ greater than control (non-sprayed plants) (Delgado et al., 2005), the authors discussed that this results mainly due to enlargement of the medullar parenchyma cells.

Values of table, 2 revealed that most of the morphological studied characters did not show any significant differences due to the interaction between cultivars and different concentrations of hydrogen peroxide with the exception of two characters, only during the first season of the experiment, number of branches per plant, and plant height. It is clear from the obtained results that the concentration of $60 \mathrm{mM}$ hydrogen peroxide is generally gave higher values for the studied characters with all the items, followed by the treatment $40 \mathrm{mM}$, while the lowest values were obtained by using zero level of hydrogen peroxide. The histological data obtained by Delgado et al. (2005) illustrated that there were more starch grains in cortex and pith tissue of $\mathrm{H}_{2} \mathrm{O}_{2}$ treated stems. The authors added that $\mathrm{H}_{2} \mathrm{O}_{2}$ also increased the number and size of xylem tracheary elements in the vascular bundles and the number and the cell wall thickness of inter-fascicular fibers.

\section{A.2. Potato tuber yield and yield components}

Data of table, 3; clearly; appeared that there were significant differences between the four genetic cultivars grown for yield and its components. In this regard, the cultivar Universal gave the highest productivity over the two years of study followed by the cultivar Vivaldi. These results coincided with the cultivar Universal which giving the highest values of the average tuber weight in the study within two years of study. Different results with respect to the proportion of tuber dry matter; where, cultivar Dora gave the highest value followed with cultivar Universal during two years of experiment, while Anushka and Vivaldi cultivars were the lowest, as appears from the data of table, 3 .

Data concerning the effect of hydrogen peroxide on tuber yield and its component characters appeared, generally, that hydrogen peroxide non-spraying gave, in general, lower values for all studied traits (No. of tubers per plant, average tuber weight, tuber yield per plant and tuber dry matter percentage)._There was no effect of different concentrations to spray hydrogen peroxide on the status of the average tuber weight trait in the first season of experiment. Non-hydrogen peroxide spray gave less value to the average tuber weight compared to spray any of the concentrations of hydrogen peroxide, while there were insignificant differences between the 
levels of hydrogen peroxide sprayed $(20,40,60 \mathrm{mM})$ in effect on such character. Particularly with respect to the number of tubers per plant, it became clear that the results of spraying concentration of $60 \mathrm{mM}$ or $40 \mathrm{mM}$ of hydrogen peroxide gave highest values compared to spray concentration of $20 \mathrm{mM}$ or non-spraying with hydrogen peroxide. Spraying with hydrogen peroxide at the rate of $40 \mathrm{mM}$ gave the highest value of tuber yield per plant without significant differences with concentrations 60 and $40 \mathrm{mM}$, while the non-spraying gave the lowest value, during the year of 2009. Highest productivity was obtained as a result of spraying concentration hydrogen peroxide at $40 \mathrm{mM}$, followed by significant differences spraying concentrations of 60 and $40 \mathrm{mM}$ hydrogen peroxide, and finally non-spraying hydrogen peroxide ranked last, during the second year of study. The results of Delgado et al. (2005) showed that the mean weights and numbers of tubers per plant were not found to be significantly differed under the different $\mathrm{H}_{2} \mathrm{O}_{2}$ concentrations.

The results of the proportion of tuber dry matter showed the presence of significant differences between the various concentrations of hydrogen peroxide over the two years (Table,3), where the results showed that the spraying concentration of $40 \mathrm{~mm}$ hydrogen peroxide gave the highest percentage of tuber dry matter compared to the other concentrations, followed by spraying concentrations of $60 \mathrm{~mm}$ and $20 \mathrm{~mm}$, respectively and finally came the treatment nonspraying with hydrogen peroxide in the latter arrangement. Figure, 1 appeared that the tubers' dry matter percentage was gradually increased with increasing hydrogen peroxide up to $40 \mathrm{mM}$, while with

Table 2. Means of the studied vegetative characters of potato during both summer seasons of 2009 and 2010

\begin{tabular}{|c|c|c|c|c|c|c|c|c|c|}
\hline \multirow{2}{*}{\multicolumn{2}{|c|}{$\begin{array}{c}\text { seasons } \\
\text { Treatments }\end{array}$}} & \multicolumn{4}{|c|}{1 st 2009} & \multicolumn{4}{|c|}{2 nd 2010} \\
\hline & & $\begin{array}{c}\text { No. of } \\
\text { branches/ } \\
\text { plant }\end{array}$ & $\begin{array}{l}\text { Plant } \\
\text { length } \\
\text { (cm) }\end{array}$ & $\begin{array}{c}\text { Foliage } \\
\text { dry matter } \\
(\%)\end{array}$ & $\begin{array}{l}\text { Branch } \\
\text { thickness } \\
\text { (cm) }\end{array}$ & $\begin{array}{c}\text { No. of } \\
\text { branches } \\
\text { / plant }\end{array}$ & $\begin{array}{l}\text { Plant } \\
\text { length } \\
(\mathrm{cm})\end{array}$ & $\begin{array}{l}\text { Foliage dry } \\
\text { matter }(\%)\end{array}$ & $\begin{array}{l}\text { Branch } \\
\text { thickness } \\
\text { (cm) }\end{array}$ \\
\hline \multicolumn{10}{|c|}{ Cultivars } \\
\hline & Anushka & $3.19 \mathrm{~b}$ & $21.25 \mathrm{~b}$ & $18.147 \mathrm{a}$ & $0.835 \mathrm{a}$ & $3.76 \mathrm{~b}$ & $28.33 b$ & $17.617 \mathrm{~b}$ & $0.414 \mathrm{a}$ \\
\hline & Universal & $3.63 \mathrm{a}$ & $28.28 \mathrm{a}$ & $19.445 \mathrm{a}$ & $0.810 \mathrm{a}$ & $4.26 \mathrm{a}$ & $33.83 \mathrm{a}$ & $19.600 \mathrm{a}$ & $0.811 \mathrm{a}$ \\
\hline & Vivaldi & $3.91 \mathrm{a}$ & $21.33 \mathrm{~b}$ & $18.290 \mathrm{a}$ & $0.665 b$ & $3.82 b$ & $23.92 \mathrm{c}$ & $18.467 \mathrm{a}$ & $0.655 b$ \\
\hline & Dora & $3.89 \mathrm{a}$ & $23.11 \mathrm{~b}$ & $17.535 \mathrm{a}$ & $0.643 b$ & $3.35 \mathrm{c}$ & $22.42 \mathrm{c}$ & $17.917 \mathrm{a}$ & $0.624 \mathrm{c}$ \\
\hline \multicolumn{10}{|c|}{$\mathrm{H}_{2} \mathrm{O}_{2}$ Concentrations } \\
\hline & $60 \mathrm{mM}$ & $3.70 \mathrm{a}$ & $21.42 \mathrm{~b}$ & $19.355 \mathrm{ab}$ & $0.800 \mathrm{a}$ & $3.75 \mathrm{a}$ & $27.83 \mathrm{a}$ & $19.85 \mathrm{a}$ & $0.756 \mathrm{a}$ \\
\hline & $40 \mathrm{mM}$ & $3.19 \mathrm{~b}$ & $23.43 \mathrm{a}$ & $20.691 \mathrm{a}$ & $0.745 b$ & $3.75 \mathrm{a}$ & $26.75 \mathrm{a}$ & $20.375 a$ & $0.747 \mathrm{ab}$ \\
\hline & $20 \mathrm{mM}$ & $4.06 \mathrm{a}$ & $24.50 \mathrm{a}$ & $18.460 \mathrm{~b}$ & $0.735 \mathrm{~b}$ & $3.86 \mathrm{a}$ & $27.08 \mathrm{a}$ & $17.85 \mathrm{~b}$ & $0.725 \mathrm{ab}$ \\
\hline & Zero & $3.66 \mathrm{a}$ & $24.64 a$ & $14.910 \mathrm{c}$ & $0.675 c$ & $3.83 \mathrm{a}$ & $26.83 a$ & $15.525 \mathrm{c}$ & $0.676 \mathrm{c}$ \\
\hline \multicolumn{10}{|c|}{ Cultivars X Concentration interaction } \\
\hline \multirow{4}{*}{ 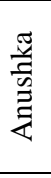 } & $60 \mathrm{mM}$ & $3.57 \mathrm{bcde}$ & $19.9 \mathrm{fgh}$ & $19.08 \mathrm{a}$ & $0.90 \mathrm{a}$ & $3.73 \mathrm{a}$ & $29.00 \mathrm{a}$ & $19.00 \mathrm{a}$ & $0.81 \mathrm{a}$ \\
\hline & $40 \mathrm{mM}$ & $2.78 \mathrm{e}$ & $18.8 \mathrm{gh}$ & $19.05 \mathrm{a}$ & $0.82 \mathrm{a}$ & $3.77 \mathrm{a}$ & $29.00 \mathrm{a}$ & $18.20 \mathrm{a}$ & $0.84 \mathrm{a}$ \\
\hline & $20 \mathrm{mM}$ & 3.00de & 22.17defg & $19.89 \mathrm{a}$ & $0.82 \mathrm{a}$ & $3.53 \mathrm{a}$ & $27.33 \mathrm{a}$ & $18.20 \mathrm{a}$ & $0.83 \mathrm{a}$ \\
\hline & Zero & $3.40 \mathrm{bcde}$ & 24.17 & $14.57 \mathrm{a}$ & $0.80 \mathrm{a}$ & $4.00 \mathrm{a}$ & $28.00 \mathrm{a}$ & $15.07 \mathrm{a}$ & $0.78 \mathrm{a}$ \\
\hline \multirow{4}{*}{ 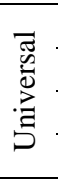 } & $60 \mathrm{mM}$ & $3.29 \mathrm{cde}$ & $28.27 \mathrm{ab}$ & $19.20 \mathrm{a}$ & $0.88 \mathrm{a}$ & $4.20 \mathrm{a}$ & $34.33 \mathrm{a}$ & $20.57 \mathrm{a}$ & $0.86 \mathrm{a}$ \\
\hline & $40 \mathrm{mM}$ & 3.18de & $28.47 \mathrm{ab}$ & $24.19 \mathrm{a}$ & $0.83 a$ & $4.23 \mathrm{a}$ & $33.00 \mathrm{a}$ & $22.53 a$ & $0.83 a$ \\
\hline & $20 \mathrm{mM}$ & $4.85 \mathrm{a}$ & $30.73 a$ & $18.50 \mathrm{a}$ & $0.82 \mathrm{a}$ & $4.57 \mathrm{a}$ & $34.00 \mathrm{a}$ & $18.50 \mathrm{a}$ & $0.81 \mathrm{a}$ \\
\hline & Zero & $3.22 \mathrm{de}$ & $25.67 \mathrm{bcd}$ & $15.89 \mathrm{a}$ & $0.72 \mathrm{a}$ & $4.00 \mathrm{a}$ & $34.00 \mathrm{a}$ & $16.80 \mathrm{a}$ & $0.76 \mathrm{a}$ \\
\hline \multirow{4}{*}{$\stackrel{i}{\frac{7}{\pi}}$} & $60 \mathrm{mM}$ & $3.63 \mathrm{bcde}$ & 19.50fgh & $20.49 \mathrm{a}$ & $0.72 \mathrm{a}$ & $3.60 \mathrm{a}$ & $24.67 \mathrm{a}$ & $20.47 \mathrm{a}$ & $0.68 \mathrm{a}$ \\
\hline & $40 \mathrm{mM}$ & $3.32 \mathrm{cde}$ & $21.94 \mathrm{efg}$ & $20.65 a$ & $0.67 \mathrm{a}$ & $3.80 \mathrm{a}$ & $22.67 \mathrm{a}$ & $20.67 \mathrm{a}$ & $0.66 \mathrm{a}$ \\
\hline & $20 \mathrm{mM}$ & $4.49 \mathrm{ab}$ & 21.37efgh & $17.84 \mathrm{a}$ & $0.66 \mathrm{a}$ & $4.00 \mathrm{a}$ & $24.00 \mathrm{a}$ & $17.67 \mathrm{a}$ & $0.66 \mathrm{a}$ \\
\hline & Zero & $4.20 \mathrm{abc}$ & 22.50def & $14.18 \mathrm{a}$ & $0.61 \mathrm{a}$ & $3.90 \mathrm{a}$ & $24.33 \mathrm{a}$ & $15.07 \mathrm{a}$ & $0.62 \mathrm{a}$ \\
\hline \multirow{4}{*}{$\stackrel{\pi}{0}$} & $60 \mathrm{mM}$ & $4.33 \mathrm{ab}$ & $18.00 \mathrm{~h}$ & $18.65 \mathrm{a}$ & $0.70 \mathrm{a}$ & $3.43 \mathrm{a}$ & $23.33 \mathrm{a}$ & $19.37 \mathrm{a}$ & $0.68 \mathrm{a}$ \\
\hline & $40 \mathrm{mM}$ & $3.50 \mathrm{bcde}$ & $24.50 \mathrm{cde}$ & $18.88 \mathrm{a}$ & $0.67 \mathrm{a}$ & $3.20 \mathrm{a}$ & $22.33 \mathrm{a}$ & $20.10 \mathrm{a}$ & $0.66 \mathrm{a}$ \\
\hline & $20 \mathrm{mM}$ & $3.91 \mathrm{bcd}$ & $23.72 \mathrm{cde}$ & $17.61 \mathrm{a}$ & $0.64 a$ & $3.33 \mathrm{a}$ & $23.00 \mathrm{a}$ & $17.03 \mathrm{a}$ & $0.60 \mathrm{a}$ \\
\hline & Zero & $3.81 \mathrm{bcd}$ & $26.22 b c$ & $15.00 \mathrm{a}$ & $0.57 \mathrm{a}$ & $3.50 \mathrm{a}$ & $21.00 \mathrm{a}$ & $15.17 \mathrm{a}$ & $0.55 \mathrm{a}$ \\
\hline
\end{tabular}

Means having an alphabetical letter in common, within a comparable group of means, do not significantly differ from each other, using Duncan's multiple range test procedure at $\mathrm{p}=0.05$ level of significance. 
Table 3. Mean performances of tuber root yield, yield components and tuber shape index of potato during both summer seasons of 2009 and 2010

\begin{tabular}{|c|c|c|c|c|c|c|c|c|c|}
\hline \multirow{2}{*}{\multicolumn{2}{|c|}{$\begin{array}{c}\text { seasons } \\
\text { Treatments }\end{array}$}} & \multicolumn{4}{|c|}{1 st 2009} & \multicolumn{4}{|c|}{2 nd 2010} \\
\hline & & $\begin{array}{c}\text { No. of } \\
\text { tubers/ } \\
\text { plant }\end{array}$ & $\begin{array}{c}\text { Average } \\
\text { tuber } \\
\text { weight } \\
\text { (gm) }\end{array}$ & $\begin{array}{l}\text { Tuber } \\
\text { yield } \\
\text { /plant } \\
(\mathrm{Kg}) \\
\end{array}$ & $\begin{array}{c}\text { Tuber } \\
\text { dry matter } \\
(\%)\end{array}$ & $\begin{array}{c}\text { No. of } \\
\text { tubers/ } \\
\text { plant }\end{array}$ & $\begin{array}{c}\text { Average } \\
\text { tuber } \\
\text { weight } \\
\text { (gm) }\end{array}$ & $\begin{array}{l}\text { Tuber yield } \\
\text { /plant }(\mathrm{Kg})\end{array}$ & $\begin{array}{c}\text { Tuber } \\
\text { dry } \\
\text { matter } \\
(\%) \\
\end{array}$ \\
\hline \multicolumn{10}{|c|}{ Cultivars } \\
\hline & Anushka & $6.89 \mathrm{c}$ & $77.22 b$ & $0.531 \mathrm{c}$ & $21.675 \mathrm{c}$ & $6.52 \mathrm{~d}$ & $96.77 \mathrm{c}$ & $0.631 \mathrm{c}$ & $21.850 \mathrm{c}$ \\
\hline & Universal & $8.44 \mathrm{bc}$ & $100.91 \mathrm{a}$ & $0.846 \mathrm{a}$ & $22.515 b$ & $9.05 \mathrm{a}$ & $136.71 \mathrm{a}$ & $1.243 \mathrm{a}$ & $22.458 \mathrm{~b}$ \\
\hline & Vivaldi & $11.39 \mathrm{a}$ & $64.64 c$ & $0.723 b$ & $21.753 \mathrm{c}$ & $7.02 \mathrm{c}$ & $115.98 b$ & $0.818 \mathrm{~b}$ & $21.816 \mathrm{c}$ \\
\hline & Dora & $9.28 \mathrm{ab}$ & $54.89 \mathrm{c}$ & $0.513 c$ & $25.910 \mathrm{a}$ & $8.13 b$ & $92.49 \mathrm{c}$ & $0.751 \mathrm{~b}$ & $25.300 \mathrm{a}$ \\
\hline \multicolumn{10}{|c|}{$\mathrm{H}_{2} \mathrm{O}_{2}$ Concentrations } \\
\hline & $60 \mathrm{mM}$ & $9.58 \mathrm{a}$ & $72.22 \mathrm{a}$ & $0.664 \mathrm{ab}$ & $23.041 \mathrm{~b}$ & $7.92 \mathrm{a}$ & $111.63 \mathrm{a}$ & $0.895 b$ & $23.214 \mathrm{~b}$ \\
\hline & $40 \mathrm{mM}$ & $9.36 \mathrm{a}$ & $78.80 \mathrm{a}$ & $0.716 \mathrm{a}$ & $23.782 \mathrm{a}$ & $8.22 \mathrm{a}$ & $115.18 \mathrm{a}$ & $0.962 \mathrm{a}$ & $23.416 \mathrm{a}$ \\
\hline & $20 \mathrm{mM}$ & $8.72 \mathrm{ab}$ & $74.82 \mathrm{a}$ & $0.642 \mathrm{ab}$ & $22.815 c$ & $7.36 \mathrm{~b}$ & $117.68 \mathrm{a}$ & $0.860 \mathrm{~b}$ & $22.558 \mathrm{c}$ \\
\hline & Zero & $8.33 \mathrm{~b}$ & $71.82 \mathrm{a}$ & $0.592 b$ & $22.214 d$ & $7.24 \mathrm{~b}$ & $97.46 \mathrm{~b}$ & $0.727 \mathrm{c}$ & $22.233 d$ \\
\hline \multicolumn{10}{|c|}{ Cultivars X Concentration interaction } \\
\hline \multirow{4}{*}{$\begin{array}{l}\frac{\pi}{y} \\
\frac{1}{5} \\
\text { 荌 }\end{array}$} & $60 \mathrm{mM}$ & $6.67 \mathrm{gh}$ & $76.80 \mathrm{a}$ & $0.511 \mathrm{a}$ & $21.45 \mathrm{gh}$ & $6.63 \mathrm{a}$ & $95.65 \mathrm{~d}$ & $0.633 \mathrm{ef}$ & $22.30 \mathrm{e}$ \\
\hline & $40 \mathrm{mM}$ & $6.44 \mathrm{~h}$ & $77.35 \mathrm{a}$ & $0.494 \mathrm{a}$ & $22.60 \mathrm{f}$ & $6.77 \mathrm{a}$ & $98.52 \mathrm{~cd}$ & $0.667 \mathrm{ef}$ & $22.23 \mathrm{e}$ \\
\hline & $20 \mathrm{mM}$ & 7.33 fgh & $86.11 \mathrm{a}$ & $0.633 \mathrm{a}$ & $21.72 \mathrm{~g}$ & $6.50 \mathrm{a}$ & $100.58 \mathrm{~cd}$ & $0.653 \mathrm{ef}$ & $21.67 \mathrm{f}$ \\
\hline & Zero & $7.11 \mathrm{fgh}$ & $68.54 \mathrm{a}$ & $0.489 \mathrm{a}$ & $21.08 \mathrm{~h}$ & $6.20 \mathrm{a}$ & $92.33 d$ & $0.573 \mathrm{f}$ & $21.20 \mathrm{~g}$ \\
\hline \multirow{4}{*}{ 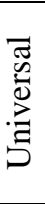 } & $60 \mathrm{mM}$ & $8.67 \mathrm{def}$ & $92.27 \mathrm{a}$ & $0.800 \mathrm{a}$ & $21.60 \mathrm{~g}$ & $9.27 \mathrm{a}$ & $140.30 \mathrm{~b}$ & $1.300 \mathrm{~b}$ & $22.17 \mathrm{e}$ \\
\hline & $40 \mathrm{mM}$ & $8.67 \mathrm{def}$ & $109.63 \mathrm{a}$ & $0.933 \mathrm{a}$ & $23.51 \mathrm{e}$ & $9.40 \mathrm{a}$ & $163.12 \mathrm{a}$ & $1.533 \mathrm{a}$ & $22.83 \mathrm{~d}$ \\
\hline & $20 \mathrm{mM}$ & $8.33 \mathrm{efg}$ & $92.89 \mathrm{a}$ & $0.772 \mathrm{a}$ & $21.57 \mathrm{~g}$ & $8.97 \mathrm{a}$ & $137.78 b$ & $1.233 b$ & $22.43 \mathrm{e}$ \\
\hline & Zero & 8.11efgh & $108.83 \mathrm{a}$ & $0.881 \mathrm{a}$ & $22.41 \mathrm{f}$ & $8.57 \mathrm{a}$ & $105.63 \mathrm{~cd}$ & $0.907 \mathrm{c}$ & $22.40 \mathrm{e}$ \\
\hline \multirow{4}{*}{ 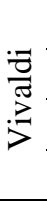 } & $60 \mathrm{mM}$ & $13.56 \mathrm{a}$ & $58.98 \mathrm{a}$ & $0.767 \mathrm{a}$ & $21.40 \mathrm{gh}$ & $7.47 \mathrm{a}$ & $114.52 \mathrm{c}$ & $0.853 \mathrm{~cd}$ & $22.17 \mathrm{e}$ \\
\hline & $40 \mathrm{mM}$ & $11.66 \mathrm{~b}$ & $71.31 \mathrm{a}$ & $0.827 \mathrm{a}$ & $22.31 \mathrm{f}$ & $7.90 \mathrm{a}$ & $108.12 \mathrm{~cd}$ & $0.850 \mathrm{~cd}$ & $22.37 \mathrm{e}$ \\
\hline & $20 \mathrm{mM}$ & $10.45 \mathrm{bcd}$ & $67.18 \mathrm{a}$ & $0.699 \mathrm{a}$ & $21.46 \mathrm{gh}$ & $5.80 \mathrm{a}$ & $141.701 \mathrm{~b}$ & $0.813 \mathrm{~cd}$ & $21.43 \mathrm{fg}$ \\
\hline & Zero & $9.89 \mathrm{bcde}$ & $61.07 \mathrm{a}$ & $0.599 \mathrm{a}$ & $21.36 \mathrm{gh}$ & $6.93 \mathrm{a}$ & $99.57 \mathrm{~cd}$ & $0.757 \mathrm{de}$ & $21.30 \mathrm{~g}$ \\
\hline \multirow{4}{*}{ 苛 } & $60 \mathrm{mM}$ & $9.45 \mathrm{cde}$ & $60.83 a$ & $0.577 \mathrm{a}$ & $27.73 a$ & $8.30 \mathrm{a}$ & $96.03 \mathrm{~cd}$ & $0.800 \mathrm{~cd}$ & $26.23 \mathrm{a}$ \\
\hline & $40 \mathrm{mM}$ & $10.67 b c$ & $56.90 \mathrm{a}$ & $0.611 \mathrm{a}$ & $27.03 \mathrm{~b}$ & $8.80 \mathrm{a}$ & $90.97 \mathrm{~d}$ & $0.800 \mathrm{~cd}$ & $26.23 \mathrm{a}$ \\
\hline & $20 \mathrm{mM}$ & 8.78def & $53.12 \mathrm{a}$ & $0.466 \mathrm{a}$ & $24.88 \mathrm{c}$ & $8.17 \mathrm{a}$ & $90.66 \mathrm{~d}$ & $0.740 \mathrm{de}$ & $24.70 \mathrm{~b}$ \\
\hline & Zero & 8.22efgh & $48.72 \mathrm{a}$ & $0.399 \mathrm{a}$ & $24.03 \mathrm{~d}$ & $7.27 \mathrm{a}$ & $92.29 d$ & $0.670 \mathrm{ef}$ & $24.03 \mathrm{c}$ \\
\hline
\end{tabular}

Means having an alphabetical letter in common, within a comparable group of means, do not significantly differ, using Duncan's multiple range test procedure at $\mathrm{p}=0.05$ level of significance.

the concentration of $60 \mathrm{mM} \mathrm{H} \mathrm{H}_{2}$, tubers' dry matter was significantly decreased through the two years of the experiment.

The examined cultivars differed in their response to spray with the four concentrations of hydrogen peroxide in a number of attributes: proportion of dry matter to tubers over two years of study; No of tubers per plant only during the first season and each of the characters average tuber weight and tuber yield per plant in the second season (Table, 3). Data of the characters: average tuber weight, tuber yield per plant (during the year of 2009) and No. of tubers per plant (during the year of 2010) showed that the studied cultivars did not differ in their responses with the differences of the concentration of hydrogen peroxide sprayed (Table, 3 ). It is, generally, appeared there was a general trend towards the exceeds of concentration $40 \mathrm{~mm}$, followed by the concentrations $60 \mathrm{~mm}$ and $20 \mathrm{~mm}$, respectively and finally non- spraying came in last order, for the three mentioned traits. 


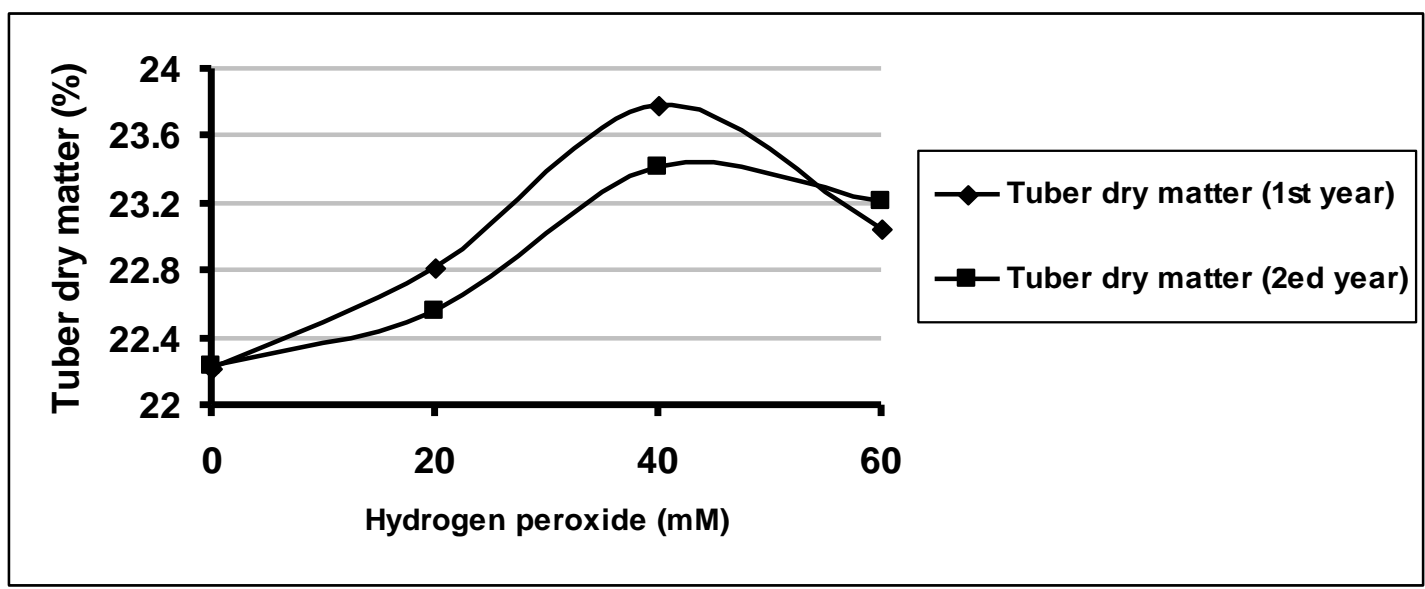

\section{Fig. 1.The relationship between tuber dry matter content $(\%)$ and hydrogen peroxide $(\mathrm{mM})$ during the two years}

\section{A.3. Potato tuber quality characteristics}

The results of table, 4 showed that the four cultivars differed among themselves with respect to tuber specific gravity and tuber starch content traits throughout the years of study. The cultivar Universal possessed the highest tuber specific gravity among the studied cultivars through the two seasons. On the other hand, there were no significant differences among the cultivars; Universal, Vivaldi and Dora for starch content in the first year while, in the second season two cultivars Universal and Dora recorded high values.

The results of the effect of hydrogen peroxide concentrations on tuber quality attributes demonstrated the following results: There were no significant differences between different concentrations of hydrogen peroxide on the status of total sugars during the two seasons. Also there was no significant effect of the concentrations of hydrogen peroxide on the status of tuber reducing sugars content during the first season only, while there were no significant differences between the concentrations of hydrogen peroxide 20,40, $60 \mathrm{mM}$ in influencing the status of tuber reducing sugars content. The two concentrations 20 and $40 \mathrm{mM}$ possessed higher significant differences values as compared to the control treatment (without spraying hydrogen peroxide). Regarding for tuber specific gravity, the results showed that although there were no significant differences between the three concentrations of hydrogen peroxide $(20,40,60 \mathrm{mM})$ in influencing on such trait in first year of study, these three concentrations gave highest significant values comparable with the control treatment. In the second year, the two treatments $60,40 \mathrm{mM}$ hydrogen peroxide gave higher specific gravity values comparison with the two treatments $20 \mathrm{mM}$ and zero hydrogen peroxide (Table, 4). Tuber starch content affected with the hydrogen peroxide where concentration of $60 \mathrm{mM}$ hydrogen peroxide produced higher starch percentage without significant differences with the concentrations of 40 and $20 \mathrm{mM}$ during the first year. The unsprayed plants (control) were the lowest in tuber starch percentage. In the second year, the concentrations of 60 and $40 \mathrm{mM}$ gave higher significant differences values when compared with the concentrations of 20 and zero $\mathrm{mM}$ of hydrogen peroxide. Figure, 2 demonstrated the previous mentioned results, where it is, clearly, appeared that tubers' starch content gradually increased with increasing the concentration of hydrogen peroxide $\left(\mathrm{H}_{2} \mathrm{O}_{2}\right)$ from zero up to $60 \mathrm{mM}$ through the two years of the study. The results obtained by Delgado et al. (2005) clearly appeared that there was significance increasing for tuber starch percentages ranged from $6.7 \%$ to $30 \%$ as a result of spraying potato plants twice-weekly with 5 or $50 \mathrm{mM} \mathrm{H} \mathrm{O}_{2}$. The authors added that, in appropriate circumstances the treatment might prove of value in commercial production, perhaps by incorporation into existing spray treatments. The authors mentioned that since the potato tuber is morphologically a modified stem, the observed starch accumulation in stems and tubers may reflect a similar cellular response.

The results of Romero and Delgado (2009) detected that treated plants with hydrogen peroxide and antioxidants such as ascorbic acid led to reduce the number of mini-tubers, while enhancing their weights and starch contents; also the leaf pigment content was increased. The authors demonstrated that hydrogen peroxide and antioxidants had positive effect in enhancing potato tuber yield and quality. Same trend of 
Table 4. Mean performances of tuber root quality attributes of potato during both summer seasons of 2009 and 2010

\begin{tabular}{|c|c|c|c|c|c|c|c|c|c|}
\hline \multirow{2}{*}{\multicolumn{2}{|c|}{$\begin{array}{c}\text { seasons } \\
\text { Treatments }\end{array}$}} & \multicolumn{4}{|c|}{1 st 2009} & \multicolumn{4}{|c|}{2 nd 2010} \\
\hline & & $\begin{array}{c}\text { Tuber } \\
\text { specific } \\
\text { gravity }\end{array}$ & $\begin{array}{c}\text { Starch } \\
(\%)\end{array}$ & $\begin{array}{c}\text { Total } \\
\text { sugars } \\
(\%)\end{array}$ & $\begin{array}{c}\text { Reducing } \\
\text { sugars } \\
(\%)\end{array}$ & $\begin{array}{l}\text { Tuber } \\
\text { specific } \\
\text { gravity }\end{array}$ & $\begin{array}{c}\text { Starch } \\
(\%)\end{array}$ & $\begin{array}{c}\text { Total } \\
\text { sugars } \\
(\%)\end{array}$ & $\begin{array}{c}\text { Reducing } \\
\text { sugars } \\
(\%)\end{array}$ \\
\hline \multicolumn{10}{|c|}{ Cultivars } \\
\hline & Anushka & $1.137 \mathrm{c}$ & $11.784 \mathrm{~b}$ & $6.595 \mathrm{a}$ & $2.183 \mathrm{a}$ & $1.142 b$ & $12.693 b$ & $6.075 \mathrm{a}$ & $2.016 \mathrm{a}$ \\
\hline & Universal & $1.223 \mathrm{a}$ & $12.604 \mathrm{a}$ & $6.457 \mathrm{a}$ & $2.220 \mathrm{a}$ & $1.225 \mathrm{a}$ & $13.300 \mathrm{a}$ & $5.991 \mathrm{a}$ & $2.050 \mathrm{a}$ \\
\hline & Vivaldi & $1.195 \mathrm{~b}$ & $12.112 \mathrm{ab}$ & $6.179 a$ & $2.112 \mathrm{a}$ & $1.133 b$ & $12.725 b$ & $6.366 \mathrm{a}$ & $2.075 \mathrm{a}$ \\
\hline & Dora & $1.133 \mathrm{c}$ & $12.325 \mathrm{ab}$ & $6.537 \mathrm{a}$ & $2.729 \mathrm{a}$ & $1.215 \mathrm{a}$ & $13.540 \mathrm{a}$ & $5.966 \mathrm{a}$ & $2.008 \mathrm{a}$ \\
\hline \multicolumn{10}{|c|}{$\mathrm{H}_{2} \mathrm{O}_{2}$ Concentrations } \\
\hline & $60 \mathrm{mM}$ & $1.195 \mathrm{a}$ & $12.620 \mathrm{a}$ & $6.300 \mathrm{a}$ & $2.187 \mathrm{a}$ & $1.192 \mathrm{a}$ & $13.541 \mathrm{a}$ & $6.016 \mathrm{a}$ & $2.025 \mathrm{ab}$ \\
\hline & $40 \mathrm{mM}$ & $1.181 \mathrm{a}$ & $12.238 \mathrm{ab}$ & $6.757 \mathrm{a}$ & $2.270 \mathrm{a}$ & $1.187 \mathrm{a}$ & $13.293 \mathrm{a}$ & $5.908 \mathrm{a}$ & $2.175 \mathrm{a}$ \\
\hline & $20 \mathrm{mM}$ & $1.174 \mathrm{a}$ & $12.116 \mathrm{ab}$ & $6.237 \mathrm{a}$ & $2.508 \mathrm{a}$ & $1.173 \mathrm{~b}$ & $12.815 b$ & $6.300 \mathrm{a}$ & $2.141 \mathrm{a}$ \\
\hline & Zero & $1.138 \mathrm{~b}$ & $11.850 \mathrm{~b}$ & $6.475 \mathrm{a}$ & $2.279 \mathrm{a}$ & $1.164 \mathrm{~b}$ & $12.607 \mathrm{~b}$ & $6.175 \mathrm{a}$ & $1.808 \mathrm{~b}$ \\
\hline \multicolumn{10}{|c|}{ Cultivars X Concentration interaction } \\
\hline \multirow{4}{*}{ 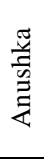 } & $60 \mathrm{mM}$ & $1.130 \mathrm{a}$ & $12.867 \mathrm{a}$ & $5.800 \mathrm{a}$ & $2.233 \mathrm{a}$ & $1.170 \mathrm{a}$ & $13.267 \mathrm{a}$ & $5.933 \mathrm{a}$ & $2.067 \mathrm{a}$ \\
\hline & $40 \mathrm{mM}$ & $1.127 \mathrm{a}$ & $11.300 \mathrm{a}$ & $7.083 \mathrm{a}$ & $2.017 \mathrm{a}$ & $1.147 \mathrm{a}$ & $13.073 \mathrm{a}$ & $5.667 \mathrm{a}$ & $2.233 \mathrm{a}$ \\
\hline & $20 \mathrm{mM}$ & $1.160 \mathrm{a}$ & $12.033 \mathrm{a}$ & $6.333 a$ & $2.167 \mathrm{a}$ & $1.140 \mathrm{a}$ & $12.033 \mathrm{a}$ & $6.333 a$ & $1.967 \mathrm{a}$ \\
\hline & Zero & $1.133 \mathrm{a}$ & $10.933 a$ & $7.167 \mathrm{a}$ & $2.317 \mathrm{a}$ & $1.113 \mathrm{a}$ & $12.400 \mathrm{a}$ & $6.367 \mathrm{a}$ & $1.800 \mathrm{a}$ \\
\hline \multirow{4}{*}{ 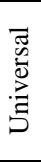 } & $60 \mathrm{mM}$ & $1.260 \mathrm{a}$ & $12.833 \mathrm{a}$ & $6.333 \mathrm{a}$ & $1.733 \mathrm{a}$ & $1.223 \mathrm{a}$ & $13.700 \mathrm{a}$ & $6.100 \mathrm{a}$ & $2.033 \mathrm{a}$ \\
\hline & $40 \mathrm{mM}$ & $1.237 \mathrm{a}$ & $13.133 \mathrm{a}$ & $6.947 \mathrm{a}$ & $2.617 \mathrm{a}$ & $1.237 \mathrm{a}$ & $13.367 \mathrm{a}$ & $6.167 \mathrm{a}$ & $2.133 \mathrm{a}$ \\
\hline & $20 \mathrm{mM}$ & $1.230 \mathrm{a}$ & $12.100 \mathrm{a}$ & $6.417 \mathrm{a}$ & $2.417 \mathrm{a}$ & $1.227 \mathrm{a}$ & $13.333 \mathrm{a}$ & $6.033 \mathrm{a}$ & $2.233 \mathrm{a}$ \\
\hline & Zero & $1.167 \mathrm{a}$ & $12.350 \mathrm{a}$ & $6.133 a$ & $2.117 \mathrm{a}$ & $1.217 \mathrm{a}$ & $12.800 \mathrm{a}$ & $5.667 \mathrm{a}$ & $1.800 \mathrm{a}$ \\
\hline \multirow{4}{*}{$\stackrel{\frac{\pi}{\pi}}{\frac{\pi}{7}}$} & $60 \mathrm{mM}$ & $1.230 \mathrm{a}$ & $11.750 \mathrm{a}$ & $6.467 \mathrm{a}$ & $2.083 \mathrm{a}$ & $1.143 \mathrm{a}$ & $13.400 \mathrm{a}$ & $6.033 \mathrm{a}$ & $2.067 \mathrm{a}$ \\
\hline & $40 \mathrm{mM}$ & $1.220 \mathrm{a}$ & $12.000 \mathrm{a}$ & $6.367 \mathrm{a}$ & $1.700 \mathrm{a}$ & $1.150 \mathrm{a}$ & $12.900 \mathrm{a}$ & $6.567 \mathrm{a}$ & $2.167 \mathrm{a}$ \\
\hline & $20 \mathrm{mM}$ & $1.183 \mathrm{a}$ & $12.450 \mathrm{a}$ & $5.733 a$ & $2.317 \mathrm{a}$ & $1.120 \mathrm{a}$ & $12.367 \mathrm{a}$ & $6.600 \mathrm{a}$ & $2.200 \mathrm{a}$ \\
\hline & Zero & $1.147 \mathrm{a}$ & $12.250 \mathrm{a}$ & $6.150 \mathrm{a}$ & $2.350 \mathrm{a}$ & $1.120 \mathrm{a}$ & $12.233 \mathrm{a}$ & $6.267 \mathrm{a}$ & $1.867 \mathrm{a}$ \\
\hline \multirow{4}{*}{ ฐ̆ } & $60 \mathrm{mM}$ & $1.160 \mathrm{a}$ & $13.033 \mathrm{a}$ & $6.600 \mathrm{a}$ & $2.700 \mathrm{a}$ & $1.233 \mathrm{a}$ & $13.800 \mathrm{a}$ & $6.000 \mathrm{a}$ & $1.933 \mathrm{a}$ \\
\hline & $40 \mathrm{mM}$ & $1.143 \mathrm{a}$ & $12.517 \mathrm{a}$ & $6.633 a$ & $2.750 \mathrm{a}$ & $1.217 \mathrm{a}$ & $13.833 \mathrm{a}$ & $5.233 \mathrm{a}$ & $2.167 \mathrm{a}$ \\
\hline & $20 \mathrm{mM}$ & $1.123 \mathrm{a}$ & $11.883 \mathrm{a}$ & $6.467 \mathrm{a}$ & $3.133 \mathrm{a}$ & $1.201 \mathrm{a}$ & $13.530 \mathrm{a}$ & $6.233 a$ & $2.167 \mathrm{a}$ \\
\hline & Zero & $1.107 \mathrm{a}$ & $11.867 \mathrm{a}$ & $6.450 \mathrm{a}$ & $2.333 \mathrm{a}$ & $1.201 \mathrm{a}$ & $12.970 \mathrm{a}$ & $6.400 \mathrm{a}$ & $1.767 \mathrm{a}$ \\
\hline
\end{tabular}

Means having an alphabetical letter in common, within a comparable group of means, do not significantly differ, using Duncan's multiple range test procedure at $\mathrm{p}=0.05$ level of significance.

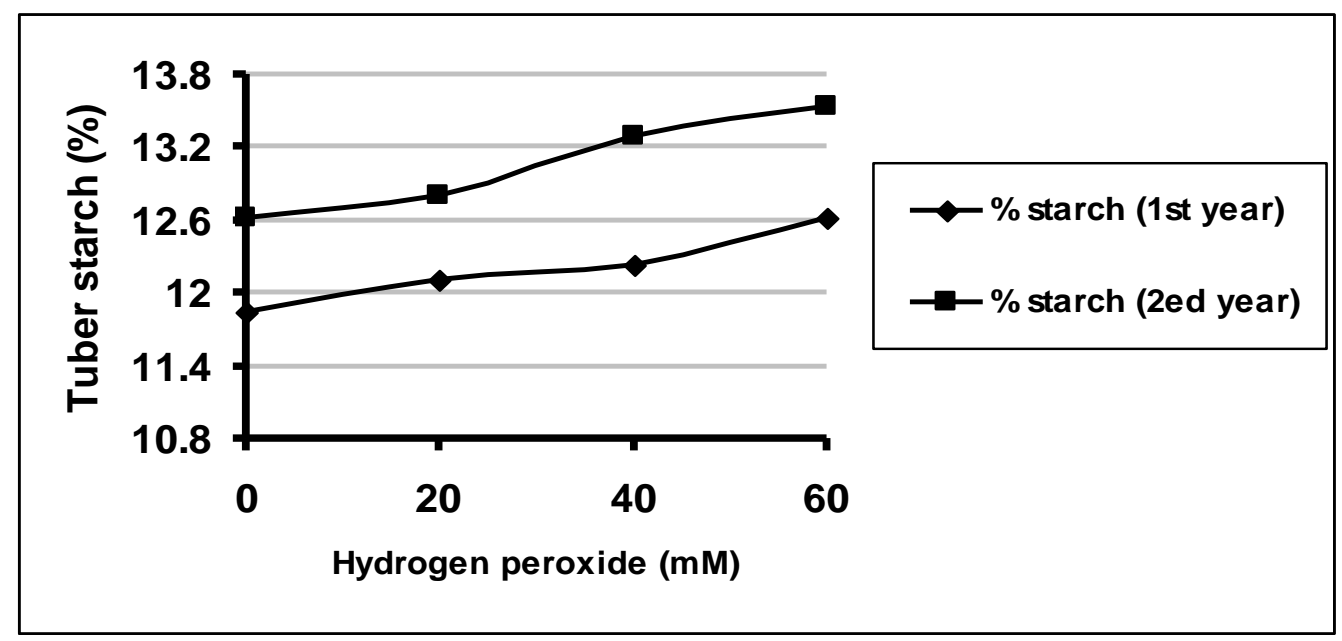

Fig. 2.The relationship between tuber starch content $(\%)$ and hydrogen peroxide $(\mathrm{mM})$ during the two years 
results were obtained by Gutierrez et al. (2012) since their results appeared that $\mathrm{H}_{2} \mathrm{O}_{2}$ treatment induced higher internal $\mathrm{H}_{2} \mathrm{O}_{2}$ concentration, which was associated with positive effects on infected mini-tubers with Phytoplasma, such as weight, reduction of number, starch content, sprouting, and tolerance to drought a signal role for $\mathrm{H}_{2} \mathrm{O}_{2}$ in lessening symptoms is suggested.

The data concerning the cultivar $\mathrm{X}$ hydrogen peroxide concentrations, clearly, appeared that none of the studied tuber quality characters showed significant differences as a result of such interactions during the two years of experiment (Table, 2). These results meant that there were same trend for the cultivar performances with spraying with the different concentrations of hydrogen peroxide. Generally, it could be conducted that spraying growing plants with the concentration of $60 \mathrm{mM}$ hydrogen peroxide gave the highest values followed by the concentrations $40 \mathrm{mM}$ and $20 \mathrm{mM}$ and finally came non-sprayed plants in the last order where the latter gave the lowest values of quality potato tubers.

\section{B. Histological data}

Data of parenchyma cell diameter are presented in table, 5. It could be conducted that the examined cultivars, significantly, differed for parenchyma cell diameter and this can be traced to genetic reasons that characterize each cultivar for the other. The results demonstrated that the cultivar Universal had the highest value where the parenchyma cell diameter recorded 211.293 micron followed with the cultivars Anushka $(203.698 \mu)$ and Vivaldi $(200.266 \mu)$ without significant differences between them while the cultivar Dora came in last place $(193.262 \mu)$. Parenchyma cell diameter was positively affected with increasing concentrations of hydrogen peroxide from zero up to $60 \mathrm{mM}$. The data detected that the ratio of increasing in tuber parenchyma cell diameter reached $9.34 \%$ as compared to non-treated plants. It could be conducted from figure, 3 that there was positive correlation between the concentration of hydrogen peroxide and the parenchyma cell diameter. It is known that starch is mainly stored in cells located parenchyma cells in tubers and thus increasing the diameter of parenchyma cells leads to an increasing in starch content of tubers and this has a positive impact on increasing the proportion of tuber's dry matter. This result is in harmony with that obtained by Delgado et al. (2005). The authors explained that applying hydrogen peroxide led to enlarge medullar parenchyma cells.

Data of table, 5 and Figure, 4 reflected that the different studied cultivars differently responded with the $\mathrm{h}_{2} \mathrm{O}_{2}$ concentrations. This indicates that there are some cultivars had positively responded to treatment with hydrogen peroxide, while other cultivars did not respond in same trend. The results showed that Anushka was did not respond correctly with the increasing concentrations of hydrogen peroxide while the Universal was responded much positive up to concentration of $40 \mathrm{mM}$. Only the two cultivars Vivaldi and Dora had responded correctly where the parenchyma cell diameter had increased steadily with increasing the hydrogen peroxide from zero up to $60 \mathrm{mM}$.

\section{Quality Evaluation of potato chips and strips processed}

\section{C.1. Dry matter}

The results in table, 6 and figure, 5 showed that there was a significance increasing in the dry matter, respecting to the first cultivation (2009), of Universal cultivar treated with $20 \mathrm{mM}$ of $\mathrm{H}_{2} \mathrm{O}_{2}$ when compared with control sample and with other samples treated with $40 \mathrm{mM}$ and $60 \mathrm{mM} \mathrm{H}_{2} \mathrm{O}_{2}$ concentrations. It could also be shown that the highest percentage of dry matter could be detected in the control samples of Vivaldi cultivar which was statistically significant

Our results of table, 6 and figure, 6 regarding the second cultivation (2010), showed that using 20, 40 or $60 \mathrm{mM} \mathrm{H} \mathrm{H}_{2} \mathrm{O}_{2}$ caused a significant increasing in the percentage of the dry matter for Anushka, compared with zero $\mathrm{mM} \mathrm{H} \mathrm{H}_{2} \mathrm{O}_{2}$. Using 20 or $60 \mathrm{mM}$ of $\mathrm{H}_{2} \mathrm{O}_{2}$ with Universal caused the highest dry matter percentages compared with using zero or $40 \mathrm{mM} \mathrm{H}_{2} \mathrm{O}_{2}$. The treatment $20 \mathrm{mM}$ of $\mathrm{H}_{2} \mathrm{O}_{2}$ was the best with the cultivar Dora. Vivaldi possessed the highest dry matter percentage when used 40 or $60 \mathrm{mM}$ of $\mathrm{H}_{2} \mathrm{O}_{2}$.

Our results agreed with that of Delgado et al. (2005) who sprayed field-grown potato plants twice weekly, from 21 to 90 days after planting, with 5 or $50 \mathrm{mM}$ hydrogen peroxide solutions. Relative to water-sprayed controls, the hydrogen peroxide treatments significantly enhanced tuber starch accumulation and increased dry matter by between $6.7 \%$ and $30 \%$. There have been few other reports describing physiological effects of exogenous $\mathrm{H}_{2} \mathrm{O}_{2}$ on potato tubers; Afek et al. (2000) found that tubers treated with a sprout inhibitor based on $\mathrm{H}_{2} \mathrm{O}_{2}$ showed sprouting inhibition after 6 months of storage.

Given the novelty of our observations on dry matter and starch accumulation, we can only engage in limited speculation on the underlying mechanisms, but it is clear that $\mathrm{H}_{2} \mathrm{O}_{2}$ treatment could prove an interesting tool for further research on dry matter and starch biosynthesis regulation. It may be that $\mathrm{H}_{2} \mathrm{O}_{2}$ treatment affects starch biosynthesis indirectly via effects on cellular signaling mechanisms. The treatment might generate a cellular redox imbalance, which would be capable of triggering 
Table 5. Means of parenchyma cell diameter during the summer season of 2010

\begin{tabular}{|c|c|c|}
\hline \multicolumn{3}{|c|}{ Parenchyma cell diameter $(\mu)$} \\
\hline & & Cultivars \\
\hline & Anushka & $203.698 \mathrm{~b}$ \\
\hline & Universal & $211.293 \mathrm{a}$ \\
\hline & Vivaldi & $200.266 \mathrm{~b}$ \\
\hline \multirow{2}{*}{\multicolumn{2}{|c|}{ Dora }} & $193.262 \mathrm{c}$ \\
\hline & & $\mathrm{H}_{2} \mathrm{O}_{2}$ Concentrations \\
\hline & $60 \mathrm{mM}$ & $210.908 \mathrm{a}$ \\
\hline & $40 \mathrm{mM}$ & $204.567 \mathrm{~b}$ \\
\hline & $20 \mathrm{mM}$ & $200.260 \mathrm{c}$ \\
\hline & Zero & $192.885 \mathrm{~d}$ \\
\hline \multirow{5}{*}{ 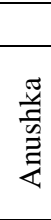 } & & Cultivars X Concentration interaction \\
\hline & $60 \mathrm{mM}$ & 197.670 ef \\
\hline & $40 \mathrm{mM}$ & $207.185 \mathrm{~d}$ \\
\hline & $20 \mathrm{mM}$ & $206.97 \mathrm{~d}$ \\
\hline & Zero & $202.895 \mathrm{de}$ \\
\hline \multirow{4}{*}{ 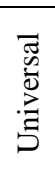 } & $60 \mathrm{mM}$ & $209.605 \mathrm{~cd}$ \\
\hline & $40 \mathrm{mM}$ & $223.025 \mathrm{a}$ \\
\hline & $20 \mathrm{mM}$ & $214.500 \mathrm{bc}$ \\
\hline & Zero & 198.000 ef \\
\hline \multirow{4}{*}{ 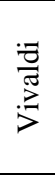 } & $60 \mathrm{mM}$ & $221.210 \mathrm{ab}$ \\
\hline & $40 \mathrm{mM}$ & $196.790 \mathrm{ef}$ \\
\hline & $20 \mathrm{mM}$ & $194.315 \mathrm{fg}$ \\
\hline & Zero & $189.145 \mathrm{gh}$ \\
\hline \multirow{4}{*}{ مَّة } & $60 \mathrm{mM}$ & $215.105 \mathrm{bc}$ \\
\hline & $40 \mathrm{mM}$ & $191.290 \mathrm{fgh}$ \\
\hline & $20 \mathrm{mM}$ & $185.185 \mathrm{hi}$ \\
\hline & Zero & $181.500 \mathrm{i}$ \\
\hline
\end{tabular}

Means having the same superscript, within a comparable group of means, do not significantly differ, using Duncan's multiple range test procedure at $\mathrm{p}=0.05$ level of significance.

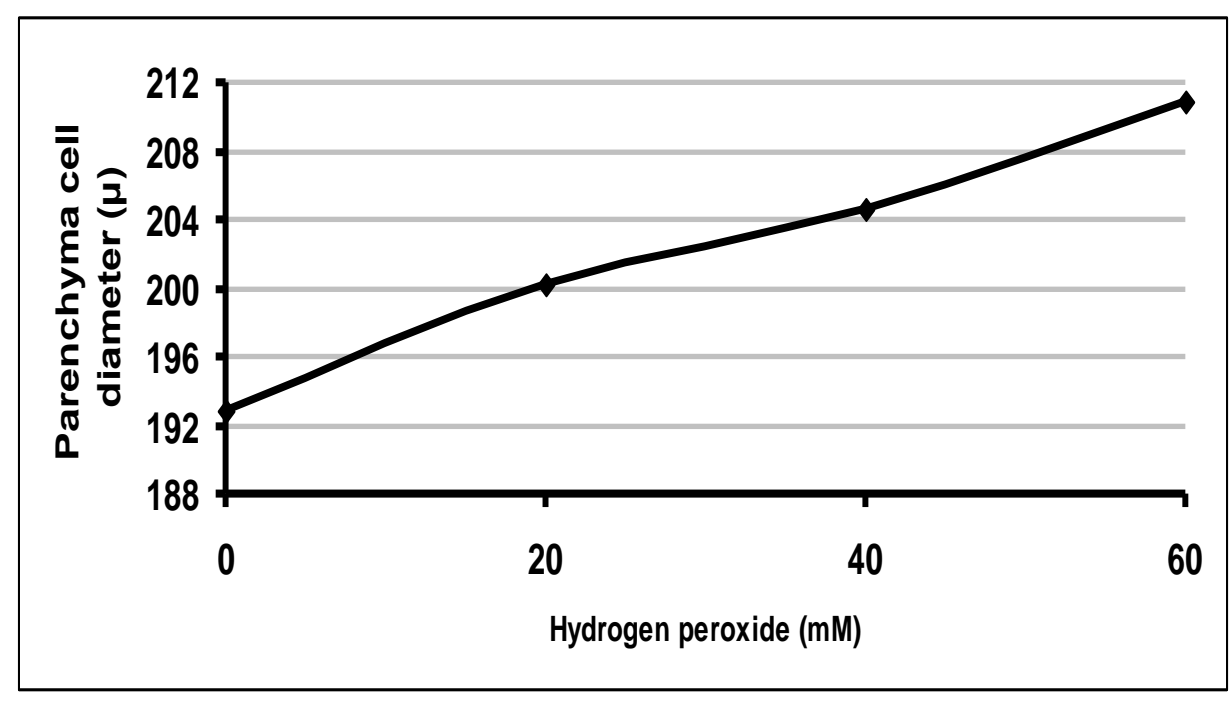

Fig. 3. The relationship between tuber parenchyma cell diameter $(\mu)$ and hydrogen peroxide (mM) 


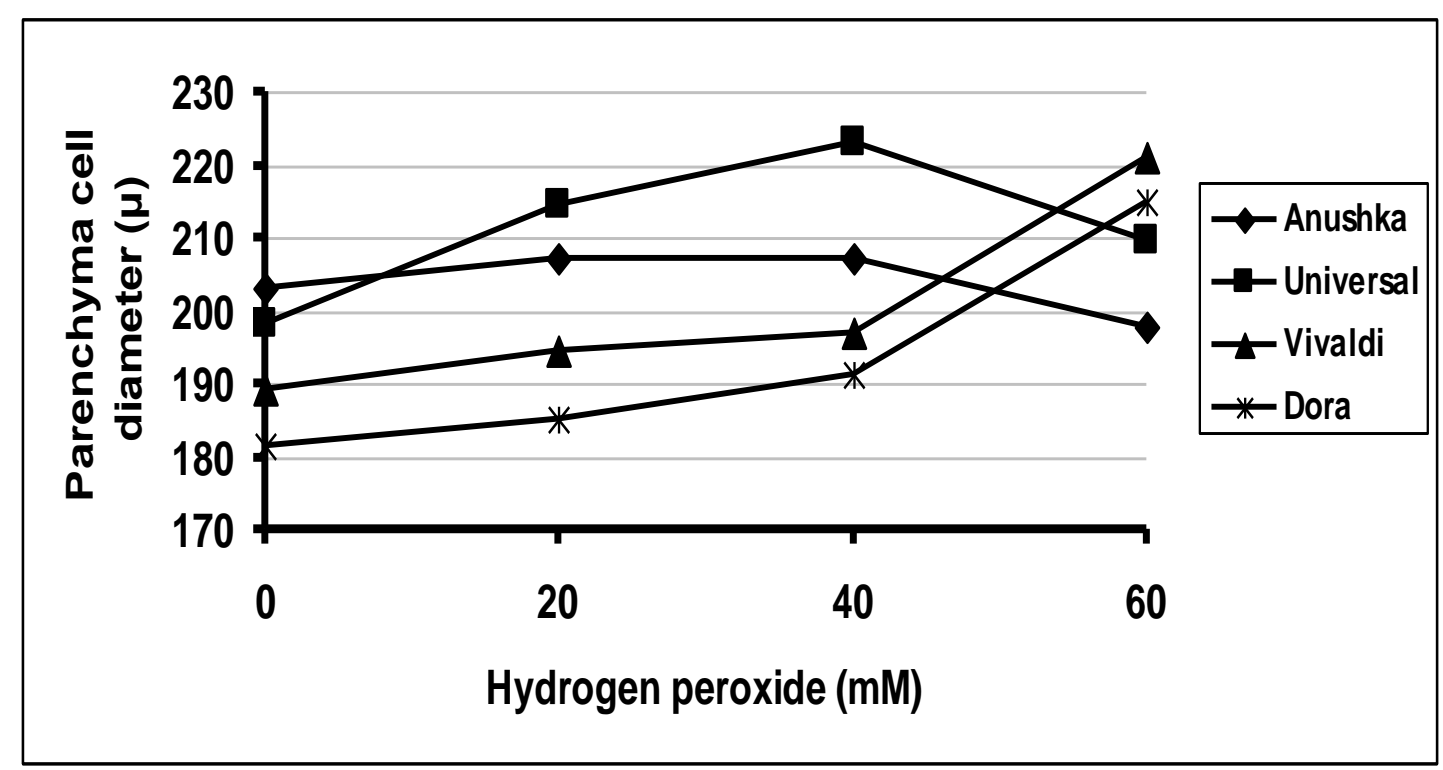

Fig. 4. The relationship between tuber parenchyma cell diameter $(\mu)$ and hydrogen peroxide (mM) for the four studied cultivars

Table 6. Dry matter percentages $(\%)$ of four potato cultivars treated with different concentrations of $\mathrm{H}_{2} \mathrm{O}_{2}$ during the first and second cultivations

\begin{tabular}{ccccccccc}
\hline \multirow{2}{\mathbf{H}_{2}\mathbf{O}_{2}}{$\begin{array}{c}\text { Season of 2009 } \\
\text { concentrations }\end{array}$} & \multicolumn{4}{c}{ Season of 2010 } \\
\cline { 2 - 8 } & Anushka & Universal & Dora & Vivaldi & Anushka & Universal & Dora & Vivaldi \\
\hline $0 \mathrm{mM}$ & $20.03^{\mathrm{a}}$ & $20.06^{\mathrm{b}}$ & $19.50^{\mathrm{a}}$ & $24.24^{\mathrm{a}}$ & $20.35^{\mathrm{b}}$ & $20.13^{\mathrm{b}}$ & $19.71^{\mathrm{b}}$ & $21.56^{\mathrm{a}}$ \\
\hline $20 \mathrm{mM}$ & $19.02^{\mathrm{a}}$ & $22.05^{\mathrm{a}}$ & $19.30^{\mathrm{a}}$ & $17.40^{\mathrm{b}}$ & $24.48^{\mathrm{a}}$ & $21.48^{\mathrm{a}}$ & $22.37^{\mathrm{a}}$ & $21.97^{\mathrm{a}}$ \\
\hline $40 \mathrm{mM}$ & $17.60^{\mathrm{a}}$ & $21.40^{\mathrm{b}}$ & $21.31^{\mathrm{a}}$ & $19.40^{\mathrm{b}}$ & $23.45^{\mathrm{a}}$ & $18.68^{\mathrm{b}}$ & $20.72^{\mathrm{b}}$ & $18.71^{\mathrm{b}}$ \\
\hline $60 \mathrm{mM}$ & $18.50^{\mathrm{a}}$ & $17.62^{\mathrm{b}}$ & $19.87^{\mathrm{a}}$ & $18.20^{\mathrm{b}}$ & $24.28^{\mathrm{a}}$ & $22.26^{\mathrm{a}}$ & $20.13^{\mathrm{b}}$ & $18.38^{\mathrm{b}}$ \\
\hline
\end{tabular}

Means having the same superscript, within a comparable group of means, do not significantly differ, using Duncan's multiple range test procedure at $\mathrm{p}=0.05$ level of significance.

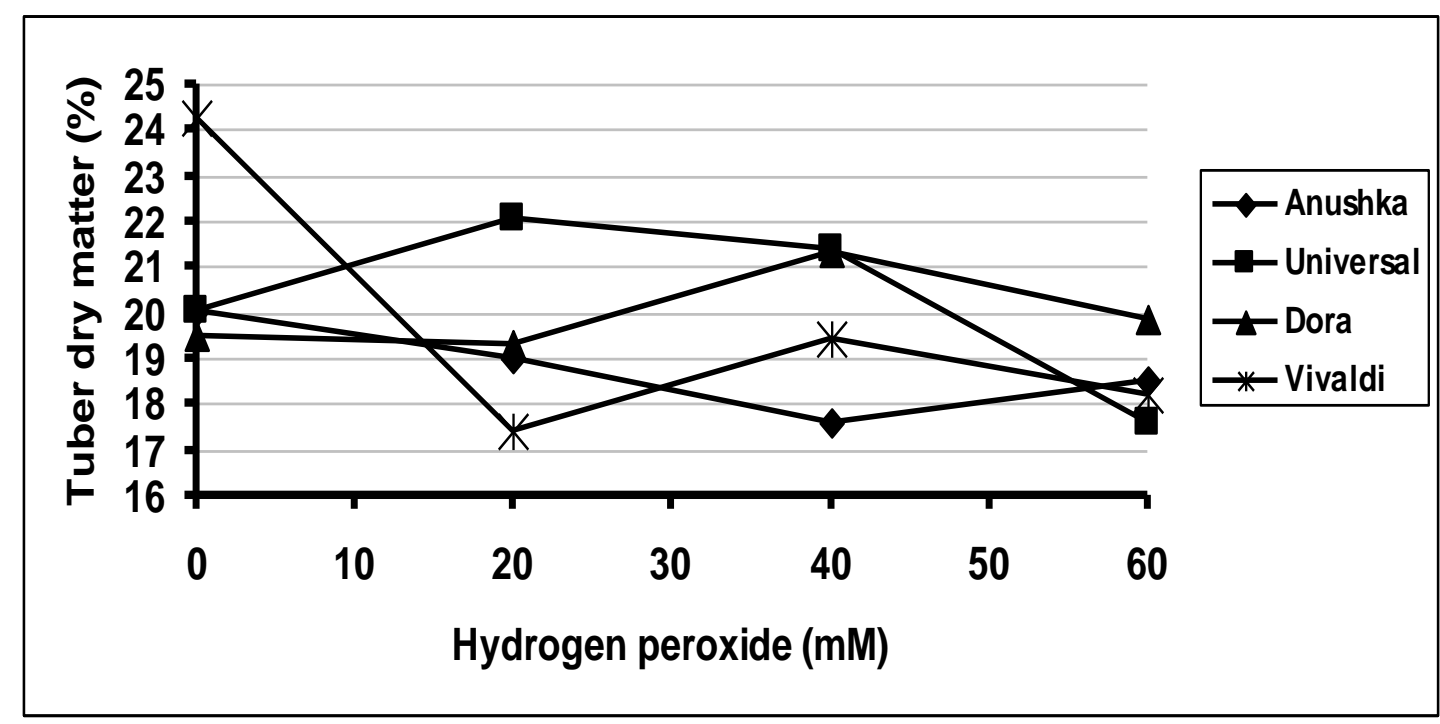

Fig. 5. Dry matter content of different potato cultivars treated with different concentrations of $\mathrm{H}_{2} \mathrm{O}_{2}$ during the first cultivation 


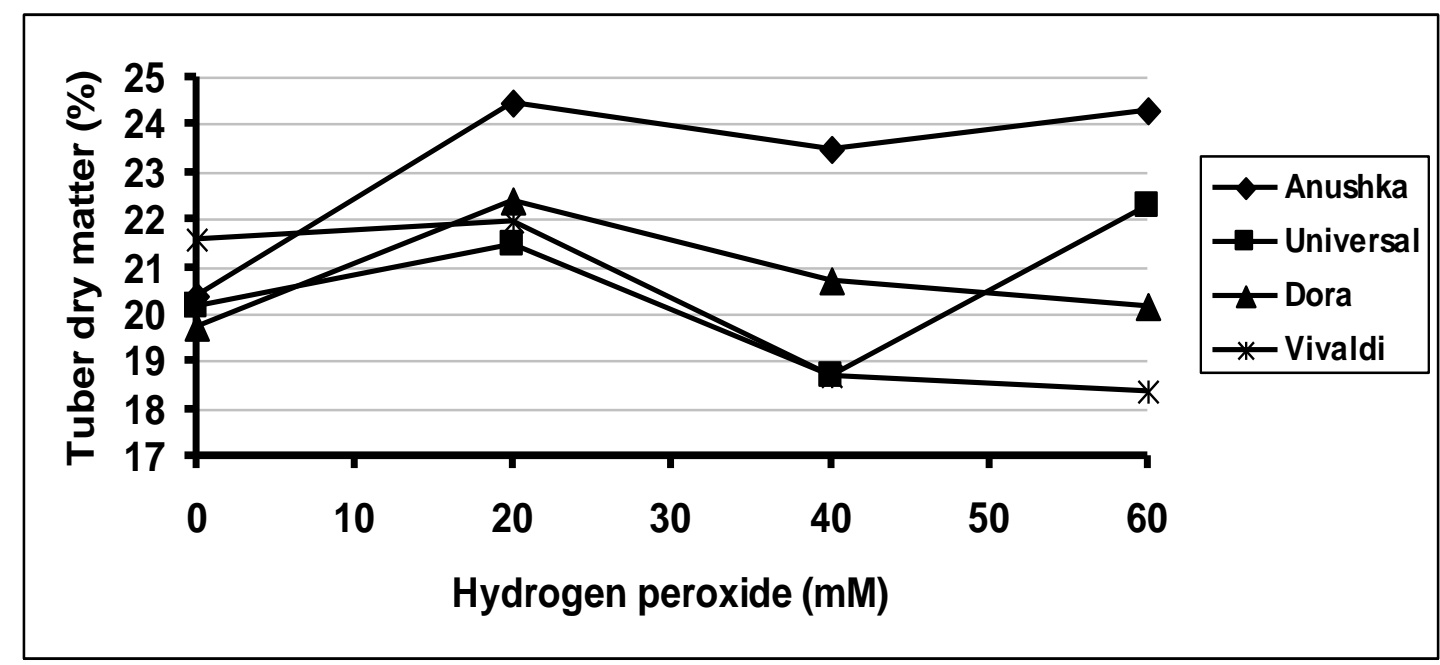

\section{Fig.6. Dry matter content of different potato cultivars treated with different concentrations of $\mathrm{H}_{2} \mathrm{O}_{2}$ during the second cultivation}

signaling cascades activating various adaptive responses, currently known examples of which include pathogen defense responses, photosynthetic adjustments, stress acclimation, and cell cycle control (Neill et al. 2002; Pastori and Foyer 2002; Dietz and Scheibe 2004).C.2. Sensory evaluation

\section{C.2.1. Potato strips (French fry) Quality}

Four primary factors determine French fry quality: dry matter, levels of reducing sugars, defects and flavor. One of the most important qualities of French fry potatoes is high dry matter, French fry processing removes water from the potato, hence the higher the water content of the potato, the more water or weight lost during processing resulting in a lower yield of fries (Talburt \& Smith, 1987). Results of table, 7, for the first year, showed that there was a significant difference in the taste attributes of Anushka variety at $0 \mathrm{mM}$ and 40 $\mathrm{mM} \mathrm{H} \mathrm{H}_{2} \mathrm{O}_{2}$ while the overall acceptability of the same variety increased significantly at $0 \mathrm{mM}$ and $20 \mathrm{mM}$ treatments. Taste values were increased significantly in

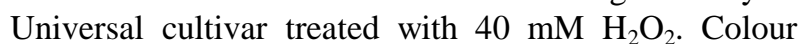
values were statistically significant at $20 \mathrm{mM}$ and 40 $\mathrm{mM} \mathrm{H} \mathrm{H}_{2} \mathrm{O}_{2}$ of Dora cultivar while the consistency value was statistically significant at $0 \mathrm{mM}$ treatment (control untreated group) of the same cultivar.

Table (7) also illustrated that values of odor attributes were statistically insignificant in all evaluated samples of the tested cultivars at different treatment concentrations.

Potatoes with high dry matter content produce a greater yield of fries than potatoes with low dry matter content. Dry matter also has a direct bearing on the amount of oil absorbed by the potato slices during the deep frying process. Fries made from tubers with low dry matter absorb more oil than fries from tubers with high dry matter. Although some oil absorption during deep frying is desirable for flavour development, too much results in limp, greasy fries. Low dry matter content increase production costs because more oil is used in the frying process (Lisińska and Gołubowska, 2005).

Basic quality characteristics of fries include: colour, taste, odor, texture and fat content (Lisiñska \& Leszczyñski, 1989). They affect the attractiveness of the ready product to a substantial extent. The results of table, 7, for the second year of the study illustrated that the taste and overall acceptability values were statistically increased for Anushka using $20 \mathrm{mM}$ of $\mathrm{H}_{2} \mathrm{O}_{2}$ followed with the treatments zero and $40 \mathrm{mM}$. For Universal cultivar, taste and overall acceptability showed significantly high values with zero, 20 and 40 $\mathrm{mM}$ of $\mathrm{H}_{2} \mathrm{O}_{2}$ compared with the treatment $60 \mathrm{mM}$.

The colour of Dora cultivar statistically possessed high significant values at $0 \mathrm{mM}$ and $40 \mathrm{mM} \mathrm{H}_{2} \mathrm{O}_{2}$ while the consistency was significantly of higher value in the control group of Dora cultivar. Colour is one of the appearance attributes of food materials, since it influences consumer acceptability (Desilkan et al., 2001). It is desirable that French fries be of a light, golden colour without any brown over colouring or black spots and traces (Lisiñska and Leszczyñski, 1989).

It can be shown from the data of table, 7 that there were not any significant differences in the mean results of sensory evaluation parameters of potato strips (French fries) produced from Vivaldi cultivar treated with the different $\mathrm{H}_{2} \mathrm{O}_{2}$ concentrations in the second cultivation. 
Table 7. Mean results of sensory evaluation of potato strips (French fries) produced from four potato cultivars treated with different $\mathrm{H}_{2} \mathrm{O}_{2}$ concentrations during the first and second cultivations

\begin{tabular}{|c|c|c|c|c|c|c|c|c|c|c|}
\hline \multirow{2}{*}{$\begin{array}{c}\text { Treatments } \\
\mathrm{H}_{2} \mathrm{O}_{2} \\
\text { concentrations }\end{array}$} & \multicolumn{5}{|c|}{ Season of 2009} & \multicolumn{5}{|c|}{ Season of 2010} \\
\hline & Colour & Odor & Taste & Consistency & Acceptability & Colour & Odor & Taste & Consistency & Acceptability \\
\hline \multicolumn{11}{|c|}{ Anushka } \\
\hline $0 \mathrm{mM}$ & $3.38 \mathrm{a}$ & $4.46 \mathrm{a}$ & $4.83 \mathrm{a}$ & $3.02 \mathrm{a}$ & $4.36 \mathrm{a}$ & $3.89^{\mathrm{a}}$ & $4.35^{\mathrm{a}}$ & $4.49^{b}$ & $3.84^{\mathrm{a}}$ & $4.36^{\mathrm{b}}$ \\
\hline $20 \mathrm{mM}$ & $3.21 \mathrm{a}$ & $4.50 \mathrm{a}$ & $4.21 \mathrm{~b}$ & $3.36 \mathrm{a}$ & $4.85 \mathrm{a}$ & $3.53^{\mathrm{a}}$ & $4.96^{\mathrm{a}}$ & $4.82^{\mathrm{a}}$ & $3.38^{\mathrm{a}}$ & $4.85^{\mathrm{a}}$ \\
\hline $40 \mathrm{mM}$ & $3.86 \mathrm{a}$ & $4.88 \mathrm{a}$ & $4.60 \mathrm{a}$ & $3.83 \mathrm{a}$ & $3.75 \mathrm{~b}$ & $3.98^{\mathrm{a}}$ & $4.28^{\mathrm{a}}$ & $4.75^{b}$ & $3.38^{\mathrm{a}}$ & $4.48^{\mathrm{b}}$ \\
\hline $60 \mathrm{mM}$ & $3.60 \mathrm{a}$ & $4.00 \mathrm{a}$ & $3.23 \mathrm{~b}$ & $3.32 \mathrm{a}$ & $4.03 \mathrm{~b}$ & $3.48^{\mathrm{a}}$ & $4.26^{\mathrm{a}}$ & $4.06^{\mathrm{c}}$ & $3.65^{\mathrm{a}}$ & $4.03^{c}$ \\
\hline \multicolumn{11}{|c|}{ Universal } \\
\hline $0 \mathrm{mM}$ & $3.31 \mathrm{a}$ & $4.15 \mathrm{a}$ & $3.87 \mathrm{~b}$ & $4.65 \mathrm{a}$ & $4.35 \mathrm{a}$ & $3.56^{\mathrm{a}}$ & $4.21^{\mathrm{a}}$ & $4.37^{\mathrm{a}}$ & $4.37^{\mathrm{a}}$ & $4.62^{\mathrm{a}}$ \\
\hline $20 \mathrm{mM}$ & $3.86 \mathrm{a}$ & $4.25 \mathrm{a}$ & $4.06 \mathrm{~b}$ & $4.26 \mathrm{a}$ & $4.22 \mathrm{a}$ & $3.21^{\mathrm{a}}$ & $4.37^{\mathrm{a}}$ & $4.61^{\mathrm{a}}$ & $4.85^{\mathrm{a}}$ & $4.24^{\mathrm{a}}$ \\
\hline $40 \mathrm{mM}$ & $3.14 \mathrm{a}$ & $4.11 \mathrm{a}$ & $4.31 \mathrm{a}$ & $4.77 \mathrm{a}$ & $4.17 \mathrm{a}$ & $3.53^{\mathrm{a}}$ & $4.41^{\mathrm{a}}$ & $4.74^{\mathrm{a}}$ & $4.35^{\mathrm{a}}$ & $4.37^{\mathrm{a}}$ \\
\hline $60 \mathrm{mM}$ & $3.75 \mathrm{a}$ & $4.39 \mathrm{a}$ & $3.75 \mathrm{~b}$ & $4.66 \mathrm{a}$ & $4.57 \mathrm{a}$ & $3.48^{\mathrm{a}}$ & $4.35^{\mathrm{a}}$ & $4.01^{b}$ & $4.39^{\mathrm{a}}$ & $4.17^{b}$ \\
\hline \multicolumn{11}{|c|}{ Dora } \\
\hline $0 \mathrm{mM}$ & $4.40 \mathrm{~b}$ & $4.47 \mathrm{a}$ & $3.00 \mathrm{a}$ & $4.50 \mathrm{a}$ & $4.04 \mathrm{a}$ & $4.64^{\mathrm{a}}$ & $4.34^{\mathrm{a}}$ & $3.17^{\mathrm{a}}$ & $4.34^{\mathrm{a}}$ & $4.11^{\mathrm{a}}$ \\
\hline $20 \mathrm{mM}$ & $4.62 \mathrm{a}$ & $4.24 \mathrm{a}$ & $3.22 \mathrm{a}$ & $3.54 \mathrm{~b}$ & $3.76 \mathrm{a}$ & $4.46^{\mathrm{b}}$ & $4.10^{\mathrm{a}}$ & $3.62^{\mathrm{a}}$ & $3.89^{b}$ & $3.28^{\mathrm{a}}$ \\
\hline $40 \mathrm{mM}$ & $4.36 \mathrm{a}$ & $4.37 \mathrm{a}$ & $3.55 \mathrm{a}$ & $3.71 \mathrm{~b}$ & $3.42 \mathrm{a}$ & $4.57^{\mathrm{a}}$ & $4.48^{\mathrm{a}}$ & $3.44^{\mathrm{a}}$ & $3.39^{\mathrm{b}}$ & $3.41^{\mathrm{a}}$ \\
\hline $60 \mathrm{mM}$ & $4.12 \mathrm{~b}$ & $4.61 \mathrm{a}$ & $3.53 \mathrm{a}$ & $3.93 \mathrm{~b}$ & $3.85 \mathrm{a}$ & $4.34^{\mathrm{b}}$ & $4.37^{\mathrm{a}}$ & $3.56^{\mathrm{a}}$ & $3.56^{\mathrm{b}}$ & $3.36^{\mathrm{a}}$ \\
\hline \multicolumn{11}{|c|}{ Vivaldi } \\
\hline $0 \mathrm{mM}$ & $3.52 \mathrm{a}$ & $4.71 \mathrm{a}$ & $3.49 \mathrm{a}$ & $3.45 \mathrm{a}$ & $4.34 \mathrm{a}$ & $3.53^{\mathrm{a}}$ & $4.46^{\mathrm{a}}$ & $3.46^{\mathrm{a}}$ & $3.72^{\mathrm{a}}$ & $4.06^{\mathrm{a}}$ \\
\hline $20 \mathrm{mM}$ & $3.64 \mathrm{a}$ & $4.56 \mathrm{a}$ & $3.19 \mathrm{a}$ & $3.30 \mathrm{a}$ & $4.02 \mathrm{a}$ & $3.82^{\mathrm{a}}$ & $4.47^{\mathrm{a}}$ & $3.29^{\mathrm{a}}$ & $3.04^{\mathrm{a}}$ & $4.43^{a}$ \\
\hline $40 \mathrm{mM}$ & $3.72 \mathrm{a}$ & $4.97 \mathrm{a}$ & $3.17 \mathrm{a}$ & $3.25 \mathrm{a}$ & $3.94 \mathrm{a}$ & $3.67^{\mathrm{a}}$ & $4.32^{\mathrm{a}}$ & $3.57^{\mathrm{a}}$ & $3.38^{\mathrm{a}}$ & $3.57^{\mathrm{a}}$ \\
\hline $60 \mathrm{mM}$ & $3.17 \mathrm{a}$ & $4.71 \mathrm{a}$ & $3.56 \mathrm{a}$ & $4.65 \mathrm{a}$ & $3.84 \mathrm{a}$ & $3.43^{\mathrm{a}}$ & $4.81^{\mathrm{a}}$ & $3.72^{\mathrm{a}}$ & $3.61^{\mathrm{a}}$ & $3.71^{\mathrm{a}}$ \\
\hline
\end{tabular}

Means having the same superscript, within a comparable group of means, do not significantly differ, using Duncan's multiple range test procedure at $\mathrm{p}=0.05$ level of significance.

5-Point descriptive category scale for each estimated parameter, where one indicating very poor and 5 indicates excellent

French fry colour is largely determined by the reducing sugars content of the potato tuber; potatoes with high reducing sugars levels make dark fries. When potatoes are fried, the reducing sugars react with amino acids in the tuber to form dark products in a nonenzymatic browning reaction. The concentration of reducing sugars in the tubers depends on cultivar, growing conditions, maturity, and storage conditions (Surmacka, 2002).

\section{C.2.2. Potato Chip Quality}

Potato chips have been popular salty snacks for 150 years and its retail sales in USA are about $\$ 6$ billion/year, representing $33 \%$ of the total sales of this market (Garayo and Moreira, 2002; and Clark, 2003).

Our results of table, 8 showed that the mean results of sensory evaluation parameters of potato chips produced from Anushka cultivar treated with different $\mathrm{H}_{2} \mathrm{O}_{2}$ concentrations in the first cultivation did not significantly differ, except that of the colour and taste. Colour, taste, consistency and overall acceptability significantly increased with the $40 \mathrm{mM} \mathrm{H}_{2} \mathrm{O}_{2}$ treatment of Universal cultivar. Dora cultivar showed a significant increasing in color, taste, consistency and overall acceptability of the control group and a significant decrease in consistency at $20 \mathrm{mM}$ and $60 \mathrm{mM} \mathrm{H}_{2} \mathrm{O}_{2}$ treatments. There was no significant difference in the Mean results of sensory evaluation of potato chips produced from Vivaldi cultivar treated with different $\mathrm{H}_{2} \mathrm{O}_{2}$ concentrations in the first cultivation except the color of the control group.

Frying in hot oil is characterized by very high water removal rates, which critically influence the mechanical; as well as, structural properties of the chips (Baumann and Escher, 1995; Hindra and Baik, 2006). The moisture content of chips decreases from around $80 \%$ to almost $2 \%$ when they are fried. However, the moisture removal inevitably leads to a considerable uptake of oil which amounts to around $35 \%$ of the mass of the chip (Aguilera and Gloria-Herna'ndez, 2000). 
Table 8. Mean results of sensory evaluation of potato chips produced from four potato cultivars treated with different $\mathrm{H}_{2} \mathrm{O}_{2}$ concentrations during the first and second cultivations.

\begin{tabular}{|c|c|c|c|c|c|c|c|c|c|c|}
\hline \multirow{2}{*}{$\begin{array}{c}\text { Treatments } \\
\mathrm{H}_{2} \mathrm{O}_{2} \\
\text { concentrations }\end{array}$} & \multicolumn{5}{|c|}{ Season of 2009} & \multicolumn{5}{|c|}{ Season of 2010} \\
\hline & Colour & Odor & Taste & Consistency & Acceptability & Colour & Odor & Taste & Consistency & Acceptability \\
\hline \multicolumn{11}{|c|}{ Anushka } \\
\hline $0 \mathrm{mM}$ & $4.00^{\mathrm{a}}$ & $4.34^{\mathrm{a}}$ & $4.07^{\mathrm{a}}$ & $3.93^{\mathrm{a}}$ & $3.95^{\mathrm{a}}$ & $3.57^{\mathrm{a}}$ & $4.76^{\mathrm{a}}$ & $4.15^{\mathrm{a}}$ & $3.76^{\mathrm{a}}$ & $4.36^{b}$ \\
\hline $20 \mathrm{mM}$ & $3.35^{b}$ & $4.23^{\mathrm{a}}$ & $3.79^{b}$ & $3.36^{\mathrm{a}}$ & $3.63^{\mathrm{a}}$ & $3.31^{\mathrm{a}}$ & $4.46^{\mathrm{a}}$ & $4.38^{\mathrm{a}}$ & $3.57^{\mathrm{a}}$ & $4.85^{\mathrm{a}}$ \\
\hline $40 \mathrm{mM}$ & $3.21^{\mathrm{b}}$ & $4.31^{\mathrm{a}}$ & $3.50^{\mathrm{b}}$ & $3.50^{\mathrm{a}}$ & $3.62 \mathrm{a}$ & $3.47^{\mathrm{a}}$ & $4.39^{a}$ & $4.02^{b}$ & $3.66^{\mathrm{a}}$ & $4.28^{c}$ \\
\hline $60 \mathrm{mM}$ & $3.36^{\mathrm{b}}$ & $4.32^{\mathrm{a}}$ & $3.64^{\mathrm{b}}$ & $3.43^{\mathrm{a}}$ & $3.43^{\mathrm{a}}$ & $3.84^{\mathrm{a}}$ & $4.43^{\mathrm{a}}$ & $4.04^{\mathrm{b}}$ & $3.73^{\mathrm{a}}$ & $4.03^{c}$ \\
\hline \multicolumn{11}{|c|}{ Universal } \\
\hline $0 \mathrm{mM}$ & $4.10^{b}$ & $4.42^{\mathrm{a}}$ & $3.90^{\mathrm{b}}$ & $3.33^{b}$ & $3.84^{b}$ & $3.23^{\mathrm{a}}$ & $4.23^{\mathrm{a}}$ & $4.54^{\mathrm{a}}$ & $4.37^{\mathrm{a}}$ & $4.39^{a}$ \\
\hline $20 \mathrm{mM}$ & $3.43^{\mathrm{b}}$ & $4.26^{\mathrm{a}}$ & $4.06^{b}$ & $3.50^{\mathrm{b}}$ & $3.81^{\mathrm{b}}$ & $3.08^{\mathrm{a}}$ & $4.75^{\mathrm{a}}$ & $4.65^{\mathrm{a}}$ & $4.85^{\mathrm{a}}$ & $4.56^{\mathrm{a}}$ \\
\hline $40 \mathrm{mM}$ & $4.44^{\mathrm{a}}$ & $4.53^{\mathrm{a}}$ & $4.31^{\mathrm{a}}$ & $4.44^{\mathrm{a}}$ & $4.43^{a}$ & $3.58^{\mathrm{a}}$ & $4.28^{\mathrm{a}}$ & $4.11^{b}$ & $4.35^{\mathrm{a}}$ & $4.41^{\mathrm{a}}$ \\
\hline $60 \mathrm{mM}$ & $3.86^{\mathrm{b}}$ & $4.62^{a}$ & $3.75^{b}$ & $3.56^{\mathrm{b}}$ & $3.72^{b}$ & $3.46^{\mathrm{a}}$ & $4.59^{\mathrm{a}}$ & $4.01^{b}$ & $4.39^{\mathrm{a}}$ & $4.17^{b}$ \\
\hline \multicolumn{11}{|c|}{ Dora } \\
\hline $0 \mathrm{mM}$ & $4.50^{\mathrm{a}}$ & $4.16^{\mathrm{a}}$ & $4.07^{\mathrm{a}}$ & $4.19^{a}$ & $4.25^{\mathrm{a}}$ & $4.64^{\mathrm{a}}$ & $4.47^{\mathrm{a}}$ & $4.52^{b}$ & $4.45^{\mathrm{a}}$ & $4.77^{\mathrm{a}}$ \\
\hline $20 \mathrm{mM}$ & $3.56^{\mathrm{b}}$ & $4.24^{\mathrm{a}}$ & $3.43^{b}$ & $2.50^{\mathrm{c}}$ & $3.43^{b}$ & $4.46^{b}$ & $4.27^{\mathrm{a}}$ & $4.63^{b}$ & $4.53^{a}$ & $4.74^{a}$ \\
\hline $40 \mathrm{mM}$ & $4.00^{b}$ & $4.04^{\mathrm{a}}$ & $3.50^{\mathrm{b}}$ & $3.19^{b}$ & $3.68^{b}$ & $4.57^{b}$ & $4.29^{\mathrm{a}}$ & $4.67^{\mathrm{a}}$ & $4.46^{\mathrm{a}}$ & $4.48^{b}$ \\
\hline $60 \mathrm{mM}$ & $3.29^{b}$ & $4.38^{\mathrm{a}}$ & $3.08^{b}$ & $2.36^{c}$ & $3.46^{\mathrm{b}}$ & $4.34^{\mathrm{b}}$ & $4.63 \mathrm{a}$ & $4.74^{\mathrm{a}}$ & $4.58^{a}$ & $4.66^{b}$ \\
\hline \multicolumn{11}{|c|}{ Vivaldi } \\
\hline $0 \mathrm{mM}$ & $4.29^{\mathrm{a}}$ & $4.28^{\mathrm{a}}$ & $3.86^{\mathrm{a}}$ & $3.86^{\mathrm{a}}$ & $4.07^{\mathrm{a}}$ & $3.32^{\mathrm{a}}$ & $4.37^{\mathrm{a}}$ & $4.09^{\mathrm{a}}$ & $3.75^{\mathrm{a}}$ & $4.30^{\mathrm{a}}$ \\
\hline $20 \mathrm{mM}$ & $3.36^{\mathrm{b}}$ & $4.10^{\mathrm{a}}$ & $3.71^{\mathrm{a}}$ & $3.36^{\mathrm{a}}$ & $3.63^{a}$ & $3.82^{\mathrm{a}}$ & $4.84^{\mathrm{a}}$ & $4.13^{\mathrm{a}}$ & $3.64^{\mathrm{a}}$ & $4.21^{\mathrm{a}}$ \\
\hline $40 \mathrm{mM}$ & $3.29^{b}$ & $4.72^{a}$ & $3.57^{\mathrm{a}}$ & $3.07^{\mathrm{a}}$ & $3.66^{\mathrm{a}}$ & $3.67^{\mathrm{a}}$ & $4.35^{\mathrm{a}}$ & $3.50^{\mathrm{b}}$ & $3.05^{\mathrm{a}}$ & $3.87^{b}$ \\
\hline $60 \mathrm{mM}$ & $4.00^{b}$ & $4.63^{a}$ & $3.50^{\mathrm{a}}$ & $2.93^{\mathrm{a}}$ & $3.48^{\mathrm{a}}$ & $3.43^{\mathrm{a}}$ & $4.41^{\mathrm{a}}$ & $3.91^{\mathrm{b}}$ & $3.48^{\mathrm{a}}$ & $3.24^{b}$ \\
\hline
\end{tabular}

Means having the same superscript, within a comparable group of means, do not significantly differ, using Duncan's multiple range test procedure at $\mathrm{p}=0.05$ level of significance.

5-Point descriptive category scale for each estimated parameter, where one indicating very poor and 5 indicates excellent.

Results of table, 8 , for the second year of the study, showed that Taste and over all acceptability significantly increased in most tested cultivars of potato chips at 0 $\mathrm{mM}$ and $20 \mathrm{mM} \mathrm{H} \mathrm{H}_{2} \mathrm{O}_{2}$ concentrations which have high dry matter percentages, except for Dora cultivar where the treatments 40 and $60 \mathrm{mM}$ possessed highest values. High dry matter is particularly important in the production of potato chips because of greater surface area to volume ratio in chips compared to fries. Chip crispness and lack of oiliness increases with increasing dry matter content (Duran et al., 2007).

Chips produced from Dora cultivar (Table, 8) showed a highly significant increasing in all tested sensory evaluation parameters at all $\mathrm{H}_{2} \mathrm{O}_{2}$ concentrations. The colour was of very good scores. Good quality potato chips have a light colour with little vascular discolouration. As with French fries, the colour of potato chips depends on the reducing sugars content of the potatoes (Biedermann-Brem, 2003). However, potato chip processors have slightly less control over

reducing sugars levels because blanching is not an option in the chipping process.

Potato chips must have a pleasing and desirable flavor, thus potatoes used in chipping must not be bitter or have other off-flavors. The flavor of potato chips is more complex than that of boiled, baked or mashed potatoes, since the cooking temperatures are higher, and the absorbed oil contributes to the overall flavor profile of the product (Bouchon et al., 2003).

\section{CONCLUSIONS}

This research is the first work in Egypt, which deals with the effect of hydrogen peroxide on the quality of potato tubers involved in potato processes. It could be concluded that the hydrogen peroxide has a good positive role in increasing the proportion of tubers' starch by increasing the breadth of parenchyma cell diameter, which are considered the main store of starch in tubers and thus increases the dry matter and then insuring increase the proportion end result of the processing. The concentration of $40 \mathrm{mM}$ hydrogen peroxide was considered as the best concentration, 
under the conditions of this study, to obtain higher percentage of tubers' dry matter. At the same time, we must recall that some of the studied cultivars might have responded much positive and better for treating with hydrogen peroxide, while some other cultivars did not respond much. So it must be doing of other researches using a greater number of cultivars to study the various aspects of the effect of hydrogen peroxide on the potato tuber quality for clear-cut conclusions. Our results demonstrated that Anushka and Universal cultivars showed highly accepted in potato strips (French fries) while Dora cultivar was the most acceptable in potato chips.

\section{REFERENCES}

Afek, U. J. Orenstein, and E. Nuriel (2000). Using HPP (Hydrogen Peroxide Plus) to inhibit potato sprouting during storage. Amer. J. Potato Res. 77:63-65.

Aguilera, J.M. and H.Gloria-Herna'ndez (2000). Oil absorption during frying of frozen parfried potatoes. Journal of Food Science 65, 446-1479.

A.O.A.C. (1970). Official Methods of Analysis Association of Official Analytical Chemists. Official and tentative methods of analysis. $11^{\text {th }}$ ed., Washington. D.C.

A.O.A.C. (1995). Official Methods of Analysis of A.O.A.C. International, $16^{\text {th }}$ edn . The Association of Analytical Chemists.

Baumann, B. and E. Escher (1995). Mass and heat transfer during deep fat frying of potato slices. Rate of drying and oil uptake. Lebensmittel- Wissenschaft und-Technologie 28, 395-403.

Biedermann-Brem (2003). How much reducing sugar may potatoes contain to avoid excessive acrylamide formation? Eur. Food Res. Technol. 217:369-373.

Bouchon, P., J.M. Aguilera and D.L. Pyle (2003). Structure oil-absorption relationships during deep-fat frying. Journal of Food Science 68, 2711-2716.

Burton W.G. (1989). The Potato, $3^{\text {ed }}$. Wiley, New York.

Clark, J.P. (2003). Happy birthday, potato chip! And other snack developments. Food Technology 57 (5), 89-92.

Delgado, L.; H.A.Z. Mancera, M.E.M. Herrera, M. V. Rivera, F. X. F. Gutiérrez and I.M. Scott (2005). Hydrogen Peroxide Increases Potato Tuber and Stem Starch Content, Stem Diameter, and Stem Lignin Content. American journal of potato research. P. 367-71

Desikan R, S.A.H. Mackerness, J.T. Hancock and S.J. Neiu (2001). Regulation of the Arabidopsis transcriptome by oxidative stress. Plant Physiol. 127:159-172.

Dietz K.J. and R. Scheibe (2004). Redox regulation: an introduction. Physiol. Plant 120:1-3.

Dubios, M, K. Gulles, J. Hamilton, P. Rebers and F. Smith (1956). Colourimetric method for determination of sugars and related substances. Analytical Chemistry. 28: 350356.
Duran, M., F. Pedreschi, P. Moyano, and E. Troncoso (2007). Oil partition in pre-treated potato slices during frying and cooling. Journal of Food Engineering 81, 257-265.

Estrada, R.N. (2000). La Biodiversidad en el Mejoramiento Gentico de la Papa. Centro International de al Papa, Lima, Pero.

Garayo, J. and R.G. Moreira (2002). Vacuum frying of potato chips. Journal of Food Engineering 55, 181-191.

Gould, W.A, and S.L. Plimpton (1985). Quality evaluation of potato cultivars for processing. Ohio Agric. Res. Dev. Cent. Res. Bull. 1172.

Gutierrez, R.M., M.E.M. Herrera and H.A.L. Delgado (2012). Genous $\mathrm{H}_{2} \mathrm{O}_{2}$ in Phytoplasma-infected potato plants promotes antioxidant activity and tuber production under drought conditions. American Journal of Potato Research, Vol. 89: 53-62.

Hindra, F. and O.D. Baik (2006). Kinetics of quality changes during food frying. Critical Reviews in Food Science and Nutrition 46, 239-258.

Lisińska, G. and G. Gołubowska (2005). Structural changes of potato tissue during French fries production. Food Chem. 93, 681-687

Lisińska G. and W. Leszczyński (1989). Potato Science and Technology, Elsevier Applied Science, London, New York., p. 391.

Neill S.J., R. Desikan, A. Clarke, R.D. Hurst and J.T. Hancock (2002). Hydrogen peroxide and nitric oxide as signalling molecules in plants. J. Exp. Bot. 53: 1237-1247.

Nickell, G.L. (1991). Proceedings $18^{\text {th }}$ Annual Meeting. Plant Growth Regulation Society of America, LaGrange, GA. Pp 115-120.

Pastori, G.M. and C.H. Foyer (2002). Common components, networks, and pathways of cross-tolerance to stress. The central role of "redox" and abscisic acid-mediated controls. Plant Physiol. 129:460-468.

Romero, R.M.T. and H.A.L. Delgado (2009). Ameliorative effects of hydrogen peroxide, ascorbate and dehydroascorbate in solanum tuberosum infected by pytoplasma. American Journal of Potato Research, Vol. 86: 218-226.

SAS Institute (2001). SAS for Windows, SAS User's Guide: Statistics.Version 8.0 e. SAS Institute Inc., Cary, NC, USA.

Snedecor, G. H. and W. C. Cochran (1980). Statistical Methods. $7^{\text {th }}$ ed. Iowa State University Press, IA, USA University press, Ames., Iowa, U.S.A.

Storey, R.MJ., and H.V. Davies (1992). Tuber quality. In: PM Harris (ed), The Potato Crop: The Scientific Basis for Improvement, $2^{\text {ed }}$. Chapman and Hall, London, pp 507569.

Surmacka, S. A. (2002). Texture is a sensory property. Food Quality Pref., 13, 215-225.

Talburt, W. F. and O. Smith (1987). Potato Processing. $\left(4^{\text {th }}\right.$ ed). Avi Van Nostrand Reinhold Company. New York, p. 796. 


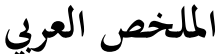

\section{دور الميدرويجين بيروكسيد في تحسين جودة درنات البطاطس}

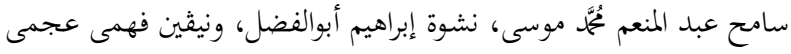

المدروسـة، وبصفة خاصـة على صفة سمـك الفـروع حيـث ازداد

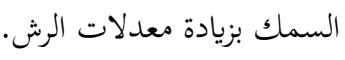

2- نباتات البطاطس التى تم رشها بالهيروجين بيروكسيد أعطت بصفة

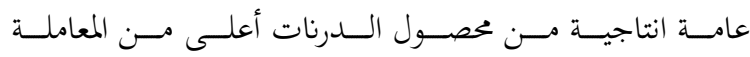

$$
\text { الكنترول(بدون رش). }
$$

3- سجلت النسبة المئوية للمادة الجافة بدرنات البطاطس أعلى نسبة لما مع المعاملة بتركيز 40 ميلليمولار وتلتها المعاملة بتركيز 60

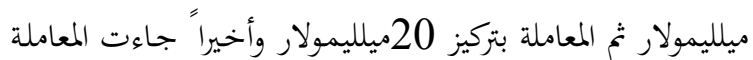

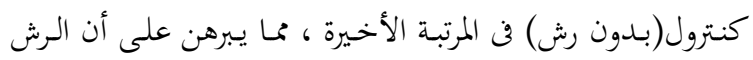
بالهيدروجين بيروكسيد ساهم بطريقة إيجابية في زيادة محتوى درنات

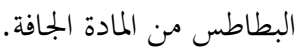
4- أوضحت النتائج أن نسبة النشا بـدرنات البطاطس قـد ازدادت

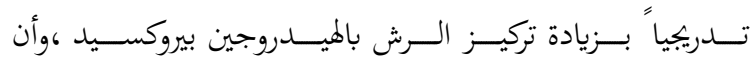

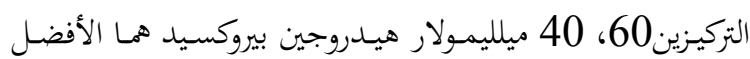

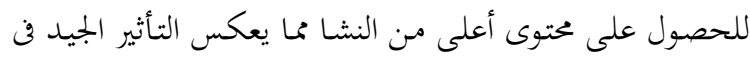
عملية تصنيع البطاطس. 5- لم يكن للهيدروجين بيروكسيد تأثير ملحوظ على محتوى درنات

$$
\text { البطاطس من السكريات الكلية. }
$$

6- بينـت إختبـارات الميسـتولوجى إزدياد قطـر الحـلايا البارانشسيمية

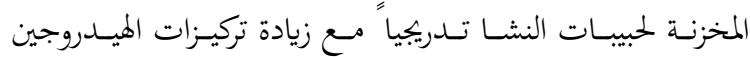
بيروكسـيد مـن صـفر وحستى تركيـز 60 ميلليمـولار بنسـبة زيادة مقدارها 34, 9\% مقارنة بالكنترول. 7- إختلفـت الأصــاف فيمـا بينهـا بالنسبة لإسـتجابتها للتركيـزات المختلفة من الهيدروجين بيروكسيد في التأثير على محتواها من المادة الجافة وذلك على مدار عامى التجربة.
أجريت هذه الدراسة خلال الموسمين الصيفيين لعامى 2009 ،

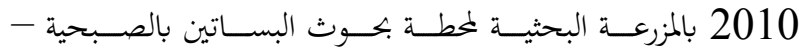

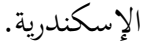

أجرى البحث بغرض دراسة تأثير مـادة الهيدروجين بيروكسيد رشاً على النباتات على زيادة جودة وتحسين درنات البطاطس الداخلة في عمليات التصنيع(الشبس، التحمير) بهدف زيادة نسبة تصافى المنتج النهائى بأقل قدر مس العيوب والذى يقلل مس قيمة المنتج النهائى

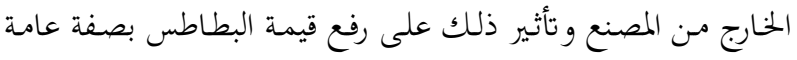

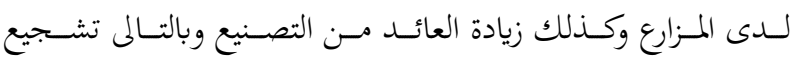

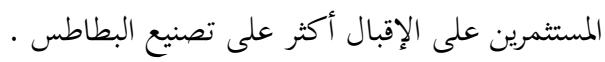

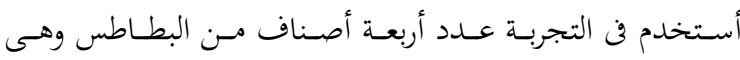

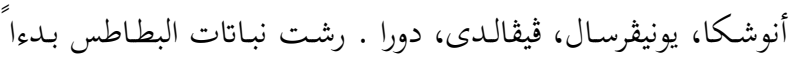

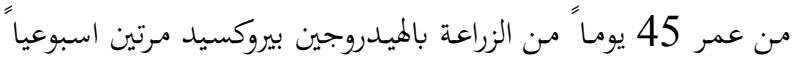
وحتى عمر 90 يوما. كانت هناك اربعة معدلات للرش بالهيدروجين

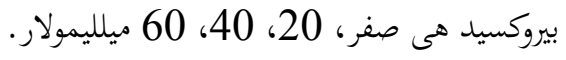

أستخدم في تطبيق التجربتين خلال السنتين نظام القطع المنشقة

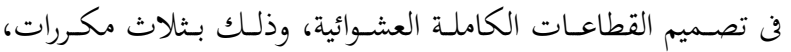
حيث وزعت عشوائيا الأصناف على القطع الرئيسية ، بينما معاملات

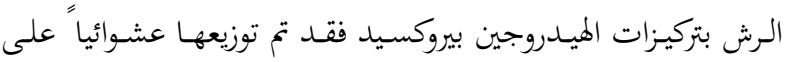

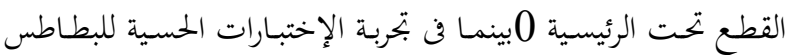
المصنعة(الأصابع المحمرة ، الشيبس) فقد أستخدم تحليل التباين وطريقة أقل فرق معنوى لدراسة الفرق بين معاملات الهيدروجين بيروكسيد الأربعة في التأثير على مزاج المتذوقين. أوضحت الدراسة النتائج التالية :1- هناك تأثير إيجابى بصفة عامة للتركيزات المرتفعة من الهيدروجين بيروكسيد(40، 60 ميلليمولار) على الصفات الخضرية 
ج- أظهرت النتائج أن الصنفين أنوشكا ، يونيثرسال هما الأفضل عند

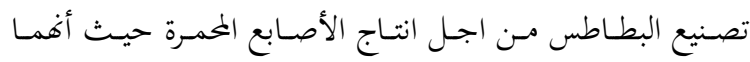

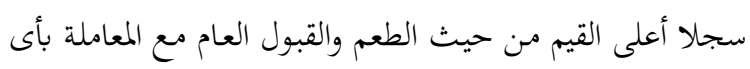

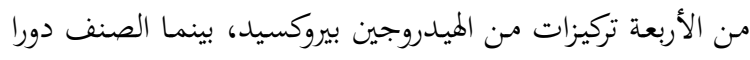

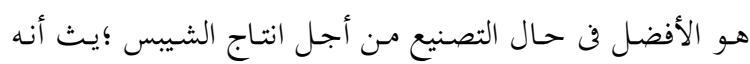

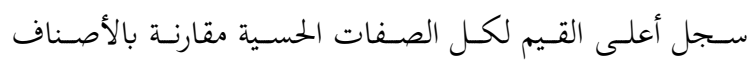
الأخرى وذلك عند جميع معاملات الهيدروجين بيروكسيد. وبناءاً على النتائج السابقة وتحت ظروف هذه التجربة فإنه يوصى

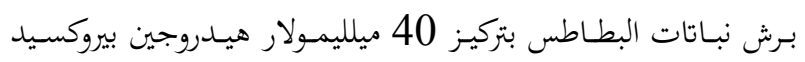

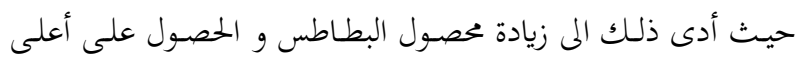
نسبة للمادة الجافة بدرنات البطاطس مما يؤدى المى زيادة نسبة تصافى ريادول

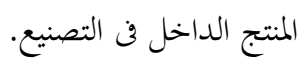

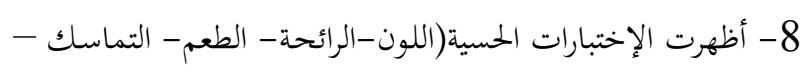

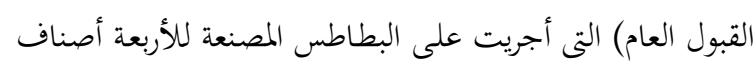

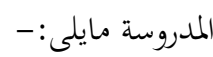
أ- عند تصنيع البطاطس من أجل إنتاج الأصابع المحمرة (المقلية) فقد

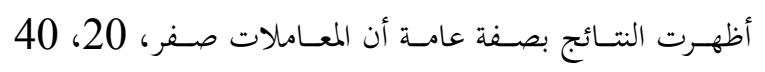
ميلليمولار هيدروجين بيروكسيد سجلت قيما أعلى بدرجة معنوية

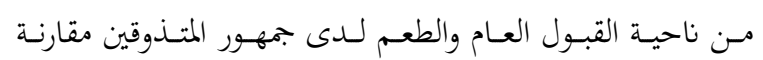

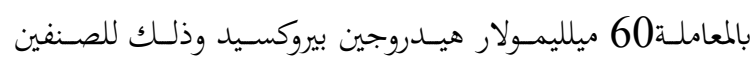
أنوشكا، و يونيثرسال. ب- عند تصنيع البطاطس من أجل إنتاج الشيبس فإن النتائج بشكل

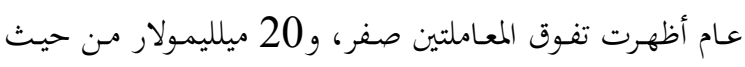

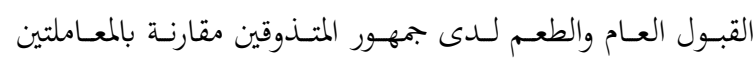

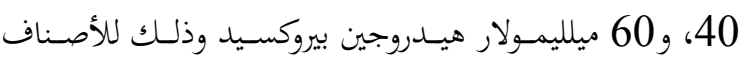

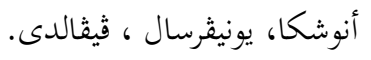

